\title{
Two-Tier Latent Class IRT Models in R
}

\author{
by Silvia Bacci and Francesco Bartolucci
}

\begin{abstract}
In analyzing data deriving from the administration of a questionnaire to a group of individuals, Item Response Theory (IRT) models provide a flexible framework to account for several aspects involved in the response process, such as the existence of multiple latent traits. In this paper, we focus on a class of semi-parametric multidimensional IRT models, in which these traits are represented through one or more discrete latent variables; these models allow us to cluster individuals into homogeneous latent classes and, at the same time, to properly study item characteristics. In particular, we follow a within-item multidimensional formulation similar to that adopted in the two-tier models, with each item measuring one or two latent traits. The proposed class of models may be estimated through the package MLCIRTwithin, whose functioning is illustrated in this paper with examples based on data about quality-of-life measurement and about the propensity to commit a crime.
\end{abstract}

\section{Introduction}

Several fields of human knowledge require the measurement of unobservable constructs (or latent traits) through ad hoc methods based on questionnaires consisting of multiple items having dichotomously or ordered politomously scored response categories. This is the case of measurement of customer satisfaction, quality-of-life, level of physical and/or psychological disabilities, ability in certain subjects, and so on.

Item Response Theory (IRT) models (Hambleton and Swaminathan, 1985; Van der Linden and Hambleton, 1997; Bartolucci et al., 2015) are well-known statistical models to deal with these data. In their original formulation, these models are characterized by: (i) unidimensionality (i.e., only one latent trait is assumed to be measured by all items); (ii) a parametric (usually normal) distribution for the latent variables used to represent the trait of interest; and (iii) no effect of individual covariates on this latent trait. These elements often turn out to be restrictive in modern applications and, therefore, several extensions of IRT models have been proposed in the literature. Among the possible extensions, in this paper we consider the class of multidimensional Latent Class (LC) IRT models proposed by Bartolucci (2007) and von Davier (2008); see also Bacci et al. (2014). Models of this type are characterized by: (i) multidimensionality, in the sense that more latent traits may be measured by the set of items (Reckase, 2009); (ii) discreteness of the latent variables, so that homogeneous subpopulations (or latent classes; Lazarsfeld and Henry, 1968; Goodman, 1974) of individuals are detected with respect to the constructs measured by the questionnaire; and (iii) possible presence of individual covariates affecting the probabilities to belong to each latent class.

In particular, we focus on a specific extension of IRT models based on within-item multidimensionality (Adams et al., 1997), which is characterized by items affected by more than one latent variable. This is opposed to the more common between-item multidimensionality, where each item may measure only one latent variable as in the original approach of Bartolucci (2007). More in detail, the model here proposed represents a discrete version of the item bifactor model and of the more general two-tier IRT model (Bock et al., 1988; Gibbons and Hedeker, 1992; Gibbons et al., 2007; Cai, 2010; Cai et al., 2011; Reise, 2012; Bonifay, 2015), based on a particular within-item multidimensional formulation with each item loading on at most two latent variables that are mutually uncorrelated. With respect to traditional item bifactor and two-tier models, which assume the normality of the latent variables, the discreteness assumption increases the flexibility of the approach and allows us to cluster individuals in homogeneous latent classes. Formann and Kohlmann (2002) propose a general approach based on latent classes that includes the discrete two-tier model here proposed as special case. However, different from the proposal of these authors, we let the class membership probability depend on individual covariates and we also allow for more flexibility in terms of specification of model link function. Limited to binary items, a recent example of application of the proposed two-tier LC-IRT model is provided in Bacci and Bartolucci (2015) to jointly study certain students' abilities and the propensity to skipping item responses.

The procedures to estimate the proposed class of two-tier LC-IRT models are implemented in the R package MLCIRTwithin (Bartolucci and Bacci, 2016), downloadable from http://CRAN. Rproject. org/package=MLCIRTwithin, whose illustration is the primary focus of the present paper. In particular, we are interested in providing a detailed description of the main functions of this package, named est_multi_poly_within and search.model_within, also through some applications.

The remainder of the paper is organized as follows. In the next section we provide the formulation of the proposed class of two-tier LC-IRT models and, then, some details about likelihood inference for these models. Furthermore, we describe the main functions implemented in the R package MLCIRTwithin for model estimation. In the following, the functioning of the package is illustrated 
through two applications: the first one concerns the measurement of Heath-related Quality Of Life (HQOL) on cancer patients and the second one is about the measurement of propensity to commit crimes. Some final remarks conclude the work.

\section{The class of models}

The proposed class of models is formulated on the basis of two independent vectors of latent variables representing the unobservable individual characteristics measured by the test items. For each unit $i=1, \ldots, n$, these vectors are denoted by $U_{i}=\left(U_{i 1}, \ldots, U_{i D_{1}}\right)^{\prime}$ and $V_{i}=\left(V_{i 1}, \ldots, V_{i D_{2}}\right)^{\prime}$ and are of dimension $D_{1}$ and $D_{2}$, respectively. Similarly to the item bifactor model, we assume that each item response $Y_{i j}$, with $i=1, \ldots, n$ and $j=1, \ldots, r$, where $r$ is the number of items, may depend on (and then measures) at most two latent variables, under the constraint that these two variables do not belong to the same vector. This is formalized by introducing the disjoint subsets $\mathcal{U}_{1}, \ldots, \mathcal{U}_{D_{1}}$ and $\mathcal{V}_{1}, \ldots, \mathcal{V}_{D_{2}}$ of $\mathcal{J}=\{1, \ldots, r\}$, where $\mathcal{U}_{d_{1}}$ contains the indices of the items depending on latent variable $U_{i d_{1}}$ and $\mathcal{V}_{d_{2}}$ is the set of those depending on latent variable $V_{i d_{2}}$. Equivalently, $Y_{i j}$ depends on $U_{i d_{1}}$ if and only if $j \in \mathcal{U}_{i d_{1}}$ and on $V_{i d_{2}}$ if and only if $j \in \mathcal{V}_{i d_{2}}$. Note that, even if the subsets $\mathcal{U}_{1}, \ldots, \mathcal{U}_{D_{1}}$ cannot overlap, and the same is assumed for $\mathcal{V}_{1}, \ldots, \mathcal{V}_{D_{2}}$, the same item $j$ may belong both to a set of the first type and to a set of the second type (within-item multidimensionality); more formally, there may exist $d_{1}$ and $d_{2}$ such that $j \in \mathcal{U}_{d_{1}}$ and $j \in \mathcal{V}_{d_{2}}$. In practice, some items belonging to $\mathcal{U}_{d_{1}}$, $d_{1}=1, \ldots, D_{1}$, will be present also in $\mathcal{V}_{d_{2}}, d_{2}=1, \ldots, D_{2}$. With respect to the specification commonly encountered in the literature on item bifactor and two-tier models, our proposal is more general, as any value of $D_{1}$ and $D_{2}$ is allowed, whereas $D_{1}=1$ (or, alternatively, $D_{2}=1$ ) in the item bifactor model and $D_{1}=2$ (or, alternatively, $D_{2}=2$ ) in the two-tier model. Moreover, components of $\boldsymbol{U}_{i}$ are allowed to be correlated; the same holds for components of $V_{i}$.

An illustrative example of the above assumptions is provided in Figure 1 , where $D_{1}=2, D_{2}=1$, and four items out of $r=7$ measure two latent traits (item 2 measures dimensions $U_{i 1}$ and $V_{i 1}$; items 3 , 5 , and 6 measure dimensions $U_{i 2}$ and $V_{i 1}$ ); the two dimensions $U_{i 1}$ and $U_{i 2}$ do not share any item.

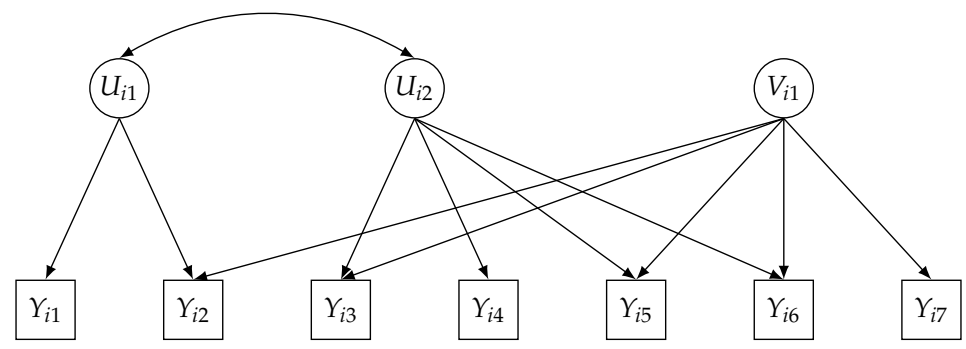

Figure 1: Path diagram of the proposed two-tier model for two latent vectors with two and one dimension, respectively, and seven items $\left(\mathcal{U}_{1}=\{1,2\}, \mathcal{U}_{2}=\{3,4,5,6\}, \mathcal{V}_{1}=\{2,3,5,6,7\}\right)$.

Adopting a semi-parametric approach for the latent distribution, the first latent vector $\boldsymbol{U}_{i}$ is assumed to have a discrete distribution based on $k_{1}$ support points $\boldsymbol{u}_{1}, \ldots, \boldsymbol{u}_{k_{1}}$ and, in absence of individual covariates, common mass probabilities $\lambda_{1}, \ldots, \lambda_{k_{1}}$. Similarly, the distribution of the second latent vector $V_{i}$ has $k_{2}$ support points $v_{1}, \ldots, v_{k_{2}}$ and, again in absence of individual covariates, common mass probabilities $\pi_{1}, \ldots, \pi_{k_{2}}$. In both cases, the support points identify classes of individuals that are homogeneous with respect to the latent traits represented by $\boldsymbol{U}_{i}$ and $\boldsymbol{V}_{i}$. Note that cases with $k_{1}=1$ or $k_{2}=1$ detect a special situation in which vector of latent variables $\boldsymbol{U}_{i}$ or $\boldsymbol{V}_{i}$, respectively, has no role in explaining the observed item responses.

For binary response variables, the measurement model assumes that, for $i=1, \ldots, n, j=1, \ldots, r$, $h_{1}=1, \ldots, k_{1}$, and $h_{2}=1, \ldots, k_{2}$,

$$
\operatorname{logit} p\left(Y_{i j}=1 \mid \boldsymbol{U}_{i}=\boldsymbol{u}_{h_{1}}, \boldsymbol{V}_{i}=\boldsymbol{v}_{h_{2}}\right)=\gamma_{1 j} \sum_{d_{1}=1}^{D_{1}} 1\left\{j \in \mathcal{U}_{d_{1}}\right\} u_{h_{1} d_{1}}+\gamma_{2 j} \sum_{d_{2}=1}^{D_{2}} 1\left\{j \in \mathcal{V}_{d_{2}}\right\} v_{h_{2} d_{2}}-\beta_{j},
$$

where $1\{\cdot\}$ is the indicator function and $\gamma_{1 j}, \gamma_{2 j}$, and $\beta_{j}$ are suitable item parameters. As usual for IRT models, $\gamma_{1 j}$ and $\gamma_{2 j}$ represent the discrimination power of item $j$ with respect to the latent variables in $\boldsymbol{U}_{i}$ and $\boldsymbol{V}_{i}$, respectively, whereas $\beta_{j}$ denotes the difficulty level of item $j$. In the previous expression, $u_{h_{1} d_{1}}$ denotes the $d_{1}$-th element of $\boldsymbol{u}_{h_{1}}$, whereas $v_{h_{2} d_{2}}$ denotes the $d_{2}$-th element of $\boldsymbol{v}_{h_{2}}$.

Different from traditional LC models characterized by constant mass probabilities, a more general approach is based on assuming that the probabilities to belong to every latent class defined by the 
distribution of $\boldsymbol{U}_{i}$ and $\boldsymbol{V}_{i}$ depend on individual covariates, when such covariates are observed. For this aim, we denote the vector of covariates for individual $i=1, \ldots, n$ by $\boldsymbol{X}_{i}$ and we assume that $\boldsymbol{U}_{i}$ and $V_{i}$ are conditionally independent given $\boldsymbol{X}_{i}$. Moreover, we adopt the following multinomial logit parametrization (Formann, 2007) for each latent vector:

$$
\begin{aligned}
& \log \frac{\lambda_{h_{1}}\left(x_{i}\right)}{\lambda_{1}\left(x_{i}\right)}=x_{i}^{\prime} \delta_{1 h_{1}}, \quad h_{1}=2, \ldots, k_{1}, \\
& \log \frac{\pi_{h_{2}}\left(x_{i}\right)}{\pi_{1}\left(x_{i}\right)}=x_{i}^{\prime} \delta_{2 h_{2}}, \quad h_{2}=2, \ldots, k_{2},
\end{aligned}
$$

with $\lambda_{h_{1}}\left(\boldsymbol{x}_{i}\right)=p\left(\boldsymbol{U}_{i}=\boldsymbol{u}_{h_{1}} \mid \boldsymbol{X}_{i}=\boldsymbol{x}_{i}\right)$ and $\pi_{h_{2}}\left(\boldsymbol{x}_{i}\right)=p\left(\boldsymbol{V}_{i}=\boldsymbol{v}_{h_{2}} \mid \boldsymbol{X}_{i}=\boldsymbol{x}_{i}\right)$, where $\boldsymbol{x}_{i}$ contains the constant term. The vectors of coefficients $\delta_{1 h_{1}}$ and $\delta_{2 h_{2}}$ measure the effect of the covariates on the logit to belong to class $h_{1}=2, \ldots, k_{1}$ and $h_{2}=2, \ldots, k_{2}$, with respect to class $h_{1}=1$ and $h_{2}=1$, respectively. Alternatively, a global logit formulation may be adopted. This is related to a cumulative logit formulation (Agresti, 2013), where the logits in equations (2) and (3) are substituted with

$$
\log \frac{p\left(\boldsymbol{U}_{i} \geq \boldsymbol{u}_{h_{1}} \mid \boldsymbol{X}_{i}=\boldsymbol{x}_{i}\right)}{p\left(\boldsymbol{U}_{i}<\boldsymbol{u}_{h_{1}} \mid \boldsymbol{X}_{i}=\boldsymbol{x}_{i}\right)}=\log \frac{\lambda_{h_{1}}\left(\boldsymbol{x}_{i}\right)+\ldots+\lambda_{k_{1}}\left(\boldsymbol{x}_{i}\right)}{\lambda_{1}\left(\boldsymbol{x}_{i}\right)+\ldots+\lambda_{h_{1}-1}\left(\boldsymbol{x}_{i}\right)}, \quad h_{1}=2, \ldots, k_{1}
$$

and

$$
\log \frac{p\left(\boldsymbol{V}_{i} \geq \boldsymbol{v}_{h_{2}} \mid \boldsymbol{X}_{i}=\boldsymbol{x}_{i}\right)}{p\left(\boldsymbol{V}_{i}<\boldsymbol{v}_{h_{2}} \mid \boldsymbol{X}_{i}=\boldsymbol{x}_{i}\right)}=\log \frac{\pi_{h_{2}}\left(\boldsymbol{x}_{i}\right)+\ldots+\pi_{k_{2}}\left(\boldsymbol{x}_{i}\right)}{\pi_{1}\left(\boldsymbol{x}_{i}\right)+\ldots+\pi_{h_{2}-1}\left(\boldsymbol{x}_{i}\right)}, \quad h_{2}=2, \ldots, k_{2},
$$

respectively. The main advantage of the global logit parametrization is the easier interpretation of the regression coefficients that now refer to the effect of the covariates on the logit to belong to a specific class (or higher) with respect to a lower class. However, this parameterization requires the latent classes to be ordered according to a specific criterion (e.g., requiring an increasing trend of the support points for a given dimension).

In the case of polytomously scored items with ordered categories indexed from 0 to $l_{j}-1$, the model based on Equation (1) may be extended according to a global logit link function, so that a graded response model (Samejima, 1969) results in:

$$
\log \frac{p\left(Y_{i j} \geq y \mid \boldsymbol{U}_{i}=\boldsymbol{u}_{h_{1}}, \boldsymbol{V}_{i}=\boldsymbol{v}_{h_{2}}\right)}{p\left(Y_{i j}<y \mid \boldsymbol{U}_{i}=\boldsymbol{u}_{h_{1}}, \boldsymbol{V}_{i}=\boldsymbol{v}_{h_{2}}\right)}=\gamma_{1 j} \sum_{d_{1}=1}^{D_{1}} 1\left\{j \in \mathcal{U}_{d_{1}}\right\} u_{h_{1} d_{1}}+\gamma_{2 j} \sum_{d_{2}=1}^{D_{2}} 1\left\{j \in \mathcal{V}_{d_{2}}\right\} v_{h_{2} d_{2}}-\beta_{j y} .
$$

Alternatively, using a local logit link function, we may assume a partial credit model (Masters, 1982):

$$
\log \frac{p\left(Y_{i j}=y \mid \boldsymbol{U}_{i}=\boldsymbol{u}_{h_{1}}, \boldsymbol{V}_{i}=\boldsymbol{v}_{h_{2}}\right)}{p\left(Y_{i j}=y-1 \mid \boldsymbol{U}_{i}=\boldsymbol{u}_{h_{1}}, \boldsymbol{V}_{i}=\boldsymbol{v}_{h_{2}}\right)}=\gamma_{1 j} \sum_{d_{1}=1}^{D_{1}} 1\left\{j \in \mathcal{U}_{d_{1}}\right\} u_{h_{1} d_{1}}+\gamma_{2 j} \sum_{d_{2}=1}^{D_{2}} 1\left\{j \in \mathcal{V}_{d_{2}}\right\} v_{h_{2} d_{2}}-\beta_{j y} .
$$

In the above expressions, $y=1, \ldots, l_{j}-1$ and the difficulty parameter $\beta_{j y}$ is now specific of item $j$ and response category $y$. A more parsimonious model is obtained by expressing $\beta_{j y}$ as the sum of two components (rating scale parametrization; Andrich, 1978), that is,

$$
\beta_{j y}=\beta_{j}+\tau_{y}, \quad j=1, \ldots, r ; y=1, \ldots, l_{j}-1,
$$

so that the distance in terms of difficulty from category to category (i.e., $\tau_{y}$ ) is the same for all items. Note that the rating scale parametrization is allowed only when items have the same number of response categories (i.e., $l_{j}=l, j=1, \ldots, r$ ). For more details about the possible item parametrizations in the presence of ordinal items see Bacci et al. (2014) and Bartolucci et al. (2015).

In order to ensure the identification of the proposed class of models, two necessary conditions must hold. First, as usual in the IRT modeling, we must constrain one discriminant index to be equal to 1 and one difficulty parameter to be equal to 0 for each dimension. More in detail, let $j_{d_{1}}$ be a specific element of $\mathcal{U}_{d_{1}}$ and $j_{d_{2}}$ a specific element of $\mathcal{V}_{d_{2}}$ for $d_{1}=1, \ldots, D_{1}$ and $d_{2}=1, \ldots, D_{2}$. Then we assume $\gamma_{1 j_{d_{1}}}=1, \gamma_{2 j_{d_{2}}}=1$, and, when item difficulties are free, $\beta_{j_{d_{1}} 1}=0$ and $\beta_{j_{d_{2}} 1}=0$, whereas in the presence of a rating scale parametrization we assume $\beta_{j_{d_{1}}}=0, \beta_{j_{d_{2}}}=0$, and $\tau_{1}=0$. In the case of binary items, constraints on difficulties simplify to $\beta_{j_{d_{1}}}=0$ and $\beta_{j_{d_{2}}}=0$. Generally speaking, $j_{d_{1}}$ and $j_{d_{2}}$ may be chosen in an arbitrary way, paying attention to select a different item for each dimension. So, in the example illustrated in Figure 1 , if we constrain item $j=1$ for dimension $U_{i 1}$ and item $j=3$ for dimension $U_{i 2}$, then for dimension $V_{i 1}$ we may constrain any one of the items in the subset $\mathcal{V}_{1}$ with the only exception of item $j=3$. As an alternative to constraining item parameters, we may fix the support points, as in the general diagnostic model of von Davier (2008).

A further identification condition requires that at least one item belongs to one of the subsets $\mathcal{U}_{d_{1}}$ or 
to one of the subsets $\mathcal{V}_{d_{2}}$; more formally, the union of $\mathcal{U}_{1}, \ldots, \mathcal{U}_{D_{1}}$ must be different from the union of $\mathcal{V}_{1}, \ldots, \mathcal{V}_{D_{2}}$. In other words, we restrict $\gamma_{1 j}=0$ or $\gamma_{2 j}=0$ for at least one $j$ and the maximum number of items shared by $\boldsymbol{U}_{i}$ and $\boldsymbol{V}_{i}$ is equal to $r-1$. Alternatively, we may skip this restrictive condition by specifying in a suitable way linear constraints (e.g., equality restrictions) on some discriminant parameters (for some examples see Cai, 2010; Cai et al., 2011).

To specify in a flexible way constraints on the support points and item parameters, we denote the complete vectors of support points by $\boldsymbol{u}=\left(u_{11}, u_{12}, \ldots, u_{k_{1} D_{1}}\right)^{\prime}$ for latent variable $\boldsymbol{U}_{i}$ and $\boldsymbol{v}=$ $\left(v_{11}, v_{12}, \ldots, v_{k_{2} D_{2}}\right)^{\prime}$ for latent variable $\boldsymbol{V}_{i}$, the complete vectors of item discriminating indices as $\gamma_{1}=$ $\left(\gamma_{11}, \ldots, \gamma_{1 r}\right)^{\prime}$ for items affected by $\boldsymbol{U}_{i}$ (then $\gamma_{1 j}$ is missing if item $j$ does not belong to $\mathcal{U}_{1}, \ldots, \mathcal{U}_{D_{1}}$ ) and $\gamma_{2}=\left(\gamma_{21}, \ldots, \gamma_{2 r}\right)^{\prime}$ for items affected by $V_{i}$ (then $\gamma_{2 j}$ is missing if item $j$ does not belong to $\left.\mathcal{V}_{1}, \ldots, \mathcal{V}_{D_{2}}\right)$, and the complete vector of item difficulties as $\beta=\left(\beta_{11}, \ldots, \beta_{r, l_{r}-1}\right)^{\prime}$ (or $\boldsymbol{\beta}=\left(\beta_{1}, \ldots, \beta_{r}\right)^{\prime}$ in the binary case). The corresponding vectors of free support points and free item parameters are denoted by $\tilde{\boldsymbol{u}}, \tilde{\boldsymbol{v}}, \tilde{\gamma}_{1}, \tilde{\gamma}_{2}$, and $\tilde{\boldsymbol{\beta}}$, respectively. A wide range of linear constraints and fixed values of the parameters are specified through a suitable definition of matrices $Z_{u}, Z_{v}, Z_{\gamma_{1}}, Z_{\gamma_{2}}$, and $Z_{\beta}$ and vectors $z_{u}, z_{v}, z_{\gamma_{1}}, z_{\gamma_{2}}$, and $z_{\beta}$, as follows:

$$
\begin{aligned}
\boldsymbol{u} & =\mathbf{Z}_{u} \tilde{\boldsymbol{u}}+z_{u}, \\
\boldsymbol{v} & =\mathbf{Z}_{v} \tilde{\boldsymbol{v}}+z_{v}, \\
\gamma_{1} & =Z_{\gamma_{1}} \tilde{\gamma}_{1}+z_{\gamma_{1}}, \\
\gamma_{2} & =Z_{\gamma_{2}} \tilde{\gamma}_{2}+z_{\gamma_{2}}, \\
\boldsymbol{\beta} & =Z_{\beta} \tilde{\boldsymbol{\beta}}+z_{\beta} .
\end{aligned}
$$

For instance, according to the usual IRT parametrization with free support points and constraints on the item parameters (i.e., one discriminant index equal to 1 and one difficulty parameter equal to 0 for each dimension), $\boldsymbol{Z}_{u}$ and $\boldsymbol{Z}_{v}$ are identity matrices of dimensions $k_{1} D_{1} \times k_{1} D_{1}$ and $k_{2} D_{2} \times k_{2} D_{2}$, respectively, and $z_{u}$ and $z_{v}$ are null vectors. Moreover, matrices $Z_{\gamma_{1}}, Z_{\gamma_{2}}$, and $Z_{\beta}$ are defined as identity matrices without those columns corresponding to the constrained item parameters, whereas $z_{\gamma_{1}}$ and $z_{\gamma_{2}}$ are vectors with ones in correspondence of constrained item discrimination parameters and zeros otherwise; $z_{\beta}$ is a vector of zeros. Further examples of specification of constraints on model parameters are provided in the sequel, when the functioning of the estimation functions of the proposed $\mathrm{R}$ package and an example on criminal data (Example 2) are illustrated.

\section{Likelihood inference}

The proposed two-tier LC-IRT model can be estimated by maximizing the marginal log-likelihood

$$
\ell(\boldsymbol{\eta})=\sum_{i=1}^{n} \log L_{i}\left(\boldsymbol{y}_{i} \mid \boldsymbol{x}_{i}\right)
$$

where $\boldsymbol{\eta}$ is the vector of free model parameters, that is, support points of $\boldsymbol{U}_{i}$ and $\boldsymbol{V}_{i}$, item difficulty and discrimination parameters, and regression coefficients for the covariates; in the previous expression, $y_{i}=\left(y_{i 1}, \ldots, y_{i r}\right)^{\prime}$ is the vector of observed item responses for subject $i$. Due to the local independence assumption, the marginal likelihood $L_{i}\left(\boldsymbol{y}_{i} \mid \boldsymbol{x}_{i}\right)$ for subject $i$ (or manifest probability of $\boldsymbol{y}_{i}$ ) used in equation (12) is given by:

$$
L_{i}\left(\boldsymbol{y}_{i} \mid \boldsymbol{x}_{i}\right)=\sum_{h_{1}=1}^{k_{1}} \sum_{h_{2}=1}^{k_{2}} \lambda_{h_{1}}\left(\boldsymbol{x}_{i}\right) \pi_{h_{2}}\left(\boldsymbol{x}_{i}\right) \prod_{j=1}^{r} p_{h_{1} h_{2}}\left(y_{i j}\right),
$$

where, $p_{h_{1} h_{2}}(y)=p\left(Y_{i j}=y \mid \boldsymbol{U}_{i}=\boldsymbol{u}_{h_{1}}, \boldsymbol{V}_{i}=\boldsymbol{v}_{h_{2}}\right)$, which depends on (1) in the case of binary items and on (4) or (5) in the case of ordinal items, if a global logit or a local logit parametrization is adopted.

We maximize $\ell(\boldsymbol{\eta})$ through the Expectation Maximization (EM) algorithm (Dempster et al., 1977), which is based on alternating two steps until convergence:

E-step: the expected value of the complete data log-likelihood (i.e., the log-likelihood for the observed and latent variables) is computed, given the current parameter vector $\eta$. In practice, this consists in computing the posterior probability $q_{h_{1} h_{2} i}$ for $h_{1}=1, \ldots, k_{1}, h_{2}=1, \ldots, k_{2}$, and $i=1, \ldots, n$; this is the probability that unit $i$ belongs to latent class $h_{1}$, according to the first vector of latent variables, and to latent class $h_{2}$, according to the second vector of latent variables, given the observed data, that is, $p\left(\boldsymbol{U}_{i}=\boldsymbol{u}_{h_{1}}, \boldsymbol{V}_{i}=\boldsymbol{v}_{h_{2}} \mid \boldsymbol{x}_{i}, \boldsymbol{y}_{i}\right)$. By the Bayes' theorem we have that

$$
q_{h_{1} h_{2} i}=\frac{\lambda_{h_{1}}\left(\boldsymbol{x}_{i}\right) \pi_{h_{2}}\left(\boldsymbol{x}_{i}\right) \prod_{j=1}^{r} p_{h_{1} h_{2}}\left(y_{i j}\right)}{L_{i}\left(\boldsymbol{y}_{i} \mid \boldsymbol{x}_{i}\right)} .
$$


The resulting complete data log-likelihood is, in expected value, equal to

$$
\ell^{*}(\boldsymbol{\eta})=\sum_{h_{1}=1}^{k_{1}} \sum_{h_{2}=1}^{k_{2}} \sum_{i=1}^{n} q_{h_{1} h_{2} i} \log \left[\lambda_{h_{1}}\left(\boldsymbol{x}_{i}\right) \pi_{h_{2}}\left(\boldsymbol{x}_{i}\right) \prod_{j=1}^{r} p_{h_{1} h_{2}}\left(y_{i j}\right)\right] .
$$

M-step: the parameter vector $\eta$ is updated by maximizing function (13) obtained at the previous step. Note that single parameter subvectors of $\eta$ may be updated separately, as this function factorizes in three components involving the mass probabilities $\lambda_{h_{1}}\left(x_{i}\right)$, the mass probabilities $\pi_{h_{2}}\left(\boldsymbol{x}_{i}\right)$, and the conditional response probabilities $p_{h_{1} h_{2}}\left(y_{i j}\right)$, respectively. Iterative algorithms of Newton-Raphson type are necessary to maximize all components (for details see Bacci and Bartolucci, 2015, and references therein) with the exception of the first two in the case of absence of individual covariates. In fact, in this case we have the following explicit expressions to update the class weights:

$$
\begin{aligned}
& \lambda_{h_{1}}=\frac{1}{n} \sum_{h_{2}=1}^{k_{2}} \sum_{i=1}^{n} q_{h_{1} h_{2} i}, \quad h_{1}=1, \ldots, k_{1}, \\
& \pi_{h_{2}}=\frac{1}{n} \sum_{h_{1}=1}^{k_{1}} \sum_{i=1}^{n} q_{h_{1} h_{2} i}, \quad h_{2}=1, \ldots, k_{2} .
\end{aligned}
$$

Similar to the other iterative algorithms, the first iteration of the EM algorithm needs to be initialized through suitable values for the model parameters that can be chosen according to certain deterministic or random rules. A common problem with finite mixture models, and then with the proposed model, is due to the presence of several local maximum points of the log-likelihood function. Therefore, in order to avoid a solution that does not correspond to the global maximum, a good practice consists in repeating the estimation process for a specific model a certain number of times using random starting values and, in the presence of different values of the log-likelihood at convergence, the solution corresponding to the highest log-likelihood value is selected.

A crucial point is that of model selection, mainly as concerns the choice of the number of support points (or latent classes) for both latent vectors (i.e., $k_{1}$ and $k_{2}$ ). For this aim, a likelihood-ratio test cannot be directly used, as the regularity conditions for having an asymptotic null distribution of $\chi^{2}$-type are not satisfied for this type of test when it is applied to compare two models with different values of $k_{1}$ and $k_{2}$. We then suggest to rely on suitable forms of penalization of the maximum log-likelihood, such as the Akaike Information Criterion (AIC; Akaike, 1973), which is related to Kullback-Leibler distance between the true density and the estimated density of a model. This criterion is based on the following index:

$$
A I C=-2 \hat{\ell}(\eta)+2 \# \text { par, }
$$

with $\hat{\ell}(\cdot)$ denoting the estimated maximum log-likelihood and \#par the number of free parameters. Alternatively, we suggest the use of the Bayesian Information Criterion (BIC; Schwarz, 1978) based on the index

$$
B I C=-2 \hat{\ell}(\boldsymbol{\eta})+\log (n) \# \text { par. }
$$

According to both these criteria, one should select the model with the minimum value of AIC or BIC.

Other selection criteria may be based on the entropy, whose computation involves the individual posterior probabilities. Entropy is a measure of the capability of the model to provide a neat partition of the sample units, which is computed as

$$
E=-\sum_{h_{1}=1}^{k_{1}} \sum_{h_{2}=1}^{k_{2}} \sum_{i=1}^{n} q_{h_{1} h_{2} i} \log q_{h_{1} h_{2} i}
$$

based on the posterior probabilities $q_{h_{1} h_{2} i}$. If the components are well separated, the posterior probabilities tend to define a clear partition of the units, assuming values close to one, and, as a consequence, the entropy will be close to zero. Usually, the entropy is not directly used to assess the number of support points and, to also account for the goodness of fit of the model, a normalized version of entropy is used by Celeux and Soromenho (1996). This is defined as

$$
N E C=\frac{E}{\hat{\ell}_{k_{1} k_{2}}-\hat{\ell}_{11}}, \quad k_{1}>1, k_{2}>1,
$$

where $\hat{\ell}_{k_{1} k_{2}}$ is the maximum log-likelihood of the model with $k_{1}$ and $k_{2}$ support points and $\hat{\ell}_{11}$ is the maximum log-likelihood value of the model with just one component for both latent variables. According to this criterion, the optimal number of components is the one that minimizes the NEC index. Note that $N E C$ is not defined when $k_{1}=k_{2}=1$, in which case $N E C=1$ by convention. 
In practice, we propose to fit a series of models with similar specifications that distinguish one other for values assigned to $k_{1}$ and $k_{2}$ and, then, to make comparisons through one or more of the mentioned criteria. In more detail, given $k_{1}$, we consider increasing values of $k_{2}$ and, similarly, given $k_{2}$ we consider increasing values of $k_{1}$ until $A I C, B I C$, or NEC do not start to increase and, then, the previous value of support points is taken as the optimal one.

More in general, for certain values of $k_{1}$ and $k_{2}$ we suggest that the choice between two competing models is driven by the likelihood-ratio test in the presence of nested models (i.e., when one model is obtained by the other one through constraints on the parameters), whereas the selection criteria above mentioned are suitable in the presence of non-nested models. The likelihood-ratio test is also used to evaluate the global fit of a model, when this is compared with the saturated model, that is, the largest model one can fit. Note that in the context at issue the saturated model is defined only for model specifications without covariates. Other proposals, coming from the literature on logistic regression models and on IRT models, consist of parametric and non-parametric tests that allow us to verify specific hypotheses concerning, among others, the unidimensionality of the questionnaire, the validity of the Rasch paradigm, the validity of the local independence assumption. In addition to the global fit of a model, also item-specific fit statistics, which are usually based on the comparison between observed and expected item responses, are useful to evaluate the goodness of each item and the need of removing it from the questionnaire. For a wide review of the mentioned methods see Bartolucci et al. (2015), Chap. 5.7, and the references therein.

Finally, in order to facilitate the interpretation of the results, we suggest to standardize the estimated support points $\hat{u}_{d_{1}} h_{1}$ and $\hat{v}_{d_{2} h_{2}}$, so as to obtain latent variables that have mean 0 and variance equal to 1 , and coherently transform the estimated item parameters $\hat{\gamma}_{1 j}, \hat{\gamma}_{2 j}$, and $\hat{\beta}_{j y}$.

Let $\hat{\mu}_{U_{d_{1}}}$ and $\hat{\sigma}_{U_{d_{1}}}$ denote the mean and the standard deviation of $\hat{u}_{d_{1} 1}, \ldots, \hat{u}_{d_{1} k_{1}}$ and let $\hat{\mu}_{V_{d_{2}}}$ and $\hat{\sigma}_{V_{d_{2}}}$ denote the mean and the standard deviation of $\hat{v}_{d_{2} 1}, \ldots, \hat{v}_{d_{2} k_{2}}$. Then, $\hat{u}_{d_{1} h_{1}}$ and $\hat{v}_{d_{2} h_{2}}$ are standardized as follows:

$$
\begin{aligned}
& \hat{u}_{d_{1} h_{1}}^{*}=\frac{\hat{u}_{d_{1} h_{1}}-\hat{\mu}_{U_{d_{1}}}}{\hat{\sigma}_{U_{d_{1}}}}, \quad d_{1}=1, \ldots, D_{1}, \\
& \hat{v}_{d_{2} h_{2}}^{*}=\frac{\hat{v}_{d_{2} h_{2}}-\hat{\mu}_{V_{d_{2}}}}{\hat{\sigma}_{V_{d_{2}}}}, \quad d_{2}=1, \ldots, D_{2} .
\end{aligned}
$$

Moreover, $\hat{\gamma}_{1 j}, \hat{\gamma}_{2 j}$, and $\hat{\beta}_{j y}$ are transformed as

$$
\begin{aligned}
& \hat{\gamma}_{1 j}^{*}=\hat{\gamma}_{1 j} \sum_{d_{1}=1}^{D_{1}} 1\left\{j \in \mathcal{U}_{d_{1}}\right\} \hat{\sigma}_{U_{d_{1}}} \\
& \hat{\gamma}_{2 j}^{*}=\hat{\gamma}_{2 j} \sum_{d_{2}=1}^{D_{2}} 1\left\{j \in \mathcal{V}_{d_{2}}\right\} \hat{\sigma}_{V_{d_{2}}} \\
& \hat{\beta}_{j y}^{*}=\hat{\beta}_{j y}-\hat{\gamma}_{1 j} \sum_{d_{1}=1}^{D_{1}} 1\left\{j \in \mathcal{U}_{d_{1}}\right\} \hat{\mu}_{U_{d_{1}}}-\hat{\gamma}_{2 j} \sum_{d_{2}=1}^{D_{2}} 1\left\{j \in \mathcal{V}_{d_{2}}\right\} \hat{\mu}_{V_{d_{2}}},
\end{aligned}
$$

for $j=1, \ldots, r$ and $y=0, \ldots, l_{j}-1$, with $l_{j}=2$ for a dichotomously scored item.

\section{The $\mathrm{R}$ package MLCIRTwithin}

The class of two-tier LC-IRT models previously described may be estimated through the R package MLCIRTwithin; for technical details see the official documentation provided in CRAN (Bartolucci and Bacci, 2016).

Before illustrating the main functions in the package at issue, it is worth mentioning some alternative $\mathrm{R}$ packages, which estimate models with a formulation resembling the one proposed. A first example is provided by the R package MultiLCIRT (Bartolucci et al., 2014, 2016), whose functions are similar, in terms of input and output, to those of MLCIRTwithin; however, MultiLCIRT is limited to the estimation of LC-IRT models under between-item multidimensionality, in the sense that items loading on more than one latent trait are not allowed and then a single vector of latent variables $\boldsymbol{U}_{i}$ is used. Moreover, constraints on the item parameters or fixed values for the support points cannot be specified. Package CDM (Robitzsch et al., 2016) performs the estimation of the class of cognitive diagnostic models (Tatsuoka, 1983; Jang, 2008; Rupp and Templin, 2008), in which the proposed, discrete, two-tier model may be included. The class of models estimated through CDM may be characterized, among the main options, by normally distributed latent traits or, alternatively, discrete 
latent traits whose support points may be freely estimated or may be specified as fixed values. As concerns item parameters, item-by-category specific slopes as well as linear constraints are allowed. However, different from our proposal, individual covariates affecting the class membership as well as the specification of a global logit link are not allowed. Moreover, attention must be paid to the interpretation of the latent classes, which are defined in a quite different and general way with respect to our proposal. The estimation of within-item multidimensional IRT models and item bifactor models is also performed through packages mirt (Chalmers, 2012; Chalmers et al., 2016) and flirt (Jeon et al., 2014), under the assumption of normally distributed latent variables. Package mirt also allows for discrete latent variables; however, in such a case just the multidimensional LC model without a classical IRT parametrization (mainly, without item difficulties) and without covariates is estimated. A major flexibility with respect to mirt is provided by package covLCA (Bertrand and Hafner, 2013), which is focused on multidimensional LC models with covariates affecting both the class membership and the manifest variables. Other two packages to mention are lavaan (Rosseel et al., 2015) and OpenMx (Neale et al., 2016) that perform the estimation of the wide class of structural equation models, in which unidimensional and multidimensional IRT models are included, under the assumption of normality of the latent variables. Finally, we mention two general and flexible softwares that may accommodate the estimation of the model here proposed, that is, Mplus (Muthén and Muthén, 2012) and LatentGold (Vermunt and Magidson, 2005): the former is tailored to the estimation of latent variable models under the assumption of normal or discrete latent variables, whereas the latter is focused on LC models. In both cases, the user may formulate IRT models with a variety of features, among which multidimensionality and presence of covariates.

\section{Functions est_multi_poly_within and est_multi_poly_between}

The main function of MLCIRTwithin is est_multi_poly_within, which performs the maximum likelihood estimation of the model specified through equations (1) to (5), allowing for several options.

Function est_multi_poly_within requires the following main input arguments:

- S: matrix of item response configurations listed row-by-row; items with a different number of categories and missing responses are allowed.

- yv: vector of the frequencies of every row in $S$; by default, $y v$ is a vector of ones.

- k1: number of latent classes for latent variable $\boldsymbol{U}_{i}$.

- k2: number of latent classes for latent variable $V_{i}$.

- $\mathrm{X}$ : matrix of covariates affecting the class weights; by default, $\mathrm{X}$ is NULL.

- start: method of initialization of the algorithm: "deterministic" (default value) for values chosen according to a deterministic rule, "random" for values randomly drawn from suitable distributions (continuous uniform between 0 and 1 for the class weights and standard normal for the other parameters), and "external" for values provided by the researcher through inputs Phi, ga1t, ga2t, De1, and De2.

- link: type of link function: "global" for global logits as in Equation (4) and "local" for local logits as in Equation (5). With binary items, any type of link function may be specified, resulting in a Rasch (Rasch, 1960) or a two-parameter logit (2PL; Birnbaum, 1968) type model depending on the value assigned to input disc.

- disc: constraints on the discriminating item parameters: FALSE (default value) for parameters $\gamma_{1 j}$ and $\gamma_{2 j}$ all equal to one and TRUE for free values. With binary items, option disc $=$ FALSE results in a Rasch model, whereas a 2PL model is obtained when option disc = TRUE.

- difl: constraints on the difficulty item parameters, in the case of ordinal polytomously scored items: FALSE (default value) for unconstrained parameters $\beta_{j y}$ and TRUE for a rating scale parametrization as in (6). This option is not allowed in the presence of items with a different number of response categories.

- multi1: matrix with one row for each component of $\boldsymbol{U}_{i}$ and elements in each cell indicating the indices of the items measuring the dimension corresponding to that row; the number of rows is $D_{1}$ and that of columns equals the number of items in the largest dimension. If dimensions differ in the number of items, zeros are inserted in the empty cells. Each item corresponding to the first column of each row has discriminating index constrained to 1 and difficulty parameter constrained to 0 to ensure model identifiability. For instance, in the presence of 6 items, with items 1 and 2 measuring the first dimension of latent variable $\boldsymbol{U}_{i}$ and the remaining items 3 to 6 measuring another dimension of $\boldsymbol{U}_{i}$, as in Figure 1, matrix multi1 is specified as

(multi1 <- $\operatorname{rbind}(c(1,2,0,0), c(3,4,5,6)))$ 


$\begin{array}{lrrrr} & {[, 1]} & {[, 2]} & {[, 3]} & {[, 4]} \\ {[1,]} & 1 & 2 & 0 & 0 \\ {[2,]} & 3 & 4 & 5 & 6\end{array}$

- multi2: same as multi1 for latent variable $\boldsymbol{V}_{i}$. For model identifiability, attention must be payed on the item indices in the first column of multi2 that cannot be the same as the indices in the first column of multi1. For instance, in the situation described in Figure 1, the matrix multi2 specified as

(multi2 $<-c(7,2: 3,5: 6))$

[1] $7 \begin{array}{lllll}7 & 2 & 3 & 5 & 6\end{array}$

implies $\gamma_{27}=1$ and $\beta_{7}=0$. A particular case is when the intersection between matrices multi 1 and multi 2 is empty: in such a case a between-item multidimensional LC-IRT model is specified, based on two completely independent latent vectors $\boldsymbol{U}_{i}$ and $\boldsymbol{V}_{i}$.

- fort: if TRUE, Fortran routines are used whenever possible to speed up computation.

- tol: level of tolerance of the algorithm in terms of relative difference between the log-likelihood corresponding to two consecutive algorithm iterations (default value is $10^{-10}$ ).

- disp: if TRUE, the log-likelihood evolution is displayed step-by-step.

- output: if TRUE, additional output arguments are returned.

- out_se: if TRUE, standard errors and variance-covariance matrix for the parameter estimates are returned.

- glob: type of parametrization for the sub-model assumed on the individual-specific latent class weights: FALSE (default value) for a multinomial logit model as in (2)-(3) and TRUE for a global logit model.

- Zth1, Zth2: matrices for the specification of linear constraints on the support points, according to (7) and (8), respectively; by default these are identity matrices with a number of rows (and columns) equal to the total number of support points, that is, $k_{1} D_{1}$ and $k_{2} D_{2}$, respectively.

- zth1, zth2: vectors of length $k_{1} D_{1}$ and $k_{2} D_{2}$, respectively, for the specification of linear constraints and fixed support points, according to (7) and (8), respectively; by default they are null vectors.

Under the default specifications of Zth1, Zth2, zth1, and zth2, the support points are freely estimated and, for the model identification, certain constraints are assumed on the item parameters. On the contrary, to fix the values of the support points, Zth1, Zth2, zth1, and zth2 must be supplied by the user. For instance, in the situation described in Figure 1 under the assumption $k_{1}=k_{2}=2$, we define $\boldsymbol{u}=(-1,-1,1,1)^{\prime}$ and $\boldsymbol{v}=(-0.5,0.5)^{\prime}$ as follows:

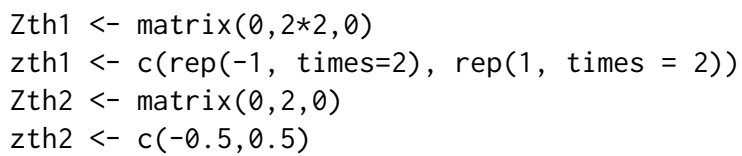

- Zga1, Zga2, Zbe: matrices for the specification of linear constraints on the vectors of item parameters $\gamma_{1}, \gamma_{2}$, and $\boldsymbol{\beta}$, as in (9), (10) and (11), respectively. In more detail, the number of rows of Zga1 and Zga2 is equal to the number of non-null entries in multi1 and multi2, respectively, and coincides with the length of vectors $\gamma_{1}$ and $\gamma_{2}$; whereas the number of rows of Zbe corresponds to the total number of item difficulties and coincides with the length of vector $\beta$. The number of columns of Zga1, Zga2, Zbe is equal to the total number of free parameters, corresponding to the length of vectors $\tilde{\gamma}_{1}, \tilde{\gamma}_{2}$, and $\tilde{\beta}$, respectively. By default these are identity matrices without those columns corresponding to the constrained parameters. For instance, in the situation described in Figure 1 with the usual IRT constraints $\gamma_{11}=\gamma_{13}=1$ and $\gamma_{27}=1$ resulting from matrices multi 1 and multi2 defined above, and $\beta_{1}=\beta_{3}=\beta_{7}=0$ in the case of binary items, the following matrices are used by default in function est_multi_poly_within:

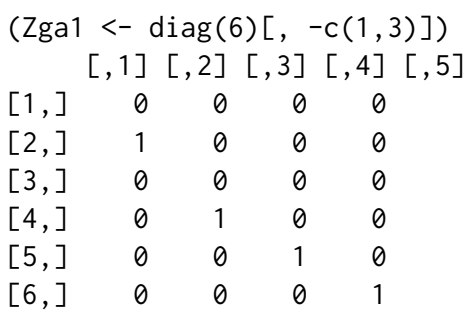


$($ Zga2 <- $\operatorname{diag}(5)[,-5])$

$\begin{array}{lrrrr} & {[, 1]} & {[, 2]} & {[, 3]} & {[, 4]} \\ {[1,]} & 1 & 0 & 0 & 0 \\ {[2,]} & 0 & 1 & 0 & 0 \\ {[3,]} & 0 & 0 & 1 & 0 \\ {[4,]} & 0 & 0 & 0 & 1 \\ {[5,]} & 0 & 0 & 0 & 0\end{array}$

(Zbe <- $\operatorname{diag}(7)[,-c(1,3,7)])$

$[, 1][, 2][, 3][, 4]$

$[1] \quad 0 \quad$,

$[2] \quad 1 \quad$,

$[3] \quad 0 \quad$,

$[4] \quad 0 \quad$,

$[5] \quad 0 \quad$,

$[6] \quad 0 \quad$,

$[7] \quad 0 \quad$,

Whenever we are interested in introducing further constraints, then the matrices at issue must be supplied by the user. For instance, to restrict $\gamma_{14}=\gamma_{15}$, then matrix Zga1 must be defined as Zga1 <- $\operatorname{diag}(6)[,-c(1,3,5)] ; \operatorname{Zga} 1[5,2]<-1$

Zga1

$[, 1][, 2][, 3]$

$[1] \quad 0 \quad$,

$[2] \quad 1 \quad 0 \quad$,

$[3] \quad 0 \quad$,

$[4] \quad 0 \quad 1 \quad$,

$[5] \quad 0 \quad 1 \quad$,

$[6] \quad 0 \quad$,

- zga1, zga2, and zbe: vectors whose length is equal to the number of rows of Zga1, Zga2, and Zbe, respectively. In other words, length of zga1, zga2, and zbe is given by the number of elements in $\gamma_{1}, \gamma_{2}$, and $\beta$. The suitable specification of these vectors, combined with that of matrices Zga1, Zga2, and Zbe, allows for linear constraints and fixed values of the item parameters, as in (9), (10), and (11). By default, zga1 and zga2 are vectors with elements 1 for each constrained item and 0 otherwise; by default zbe is a null vector. For instance, in the situation depicted in Figure 1 and matrices multi1 and multi2, default values assumed for vectors at issue are

zga1 <- c(1, $0,1,0,0,0)$

zga2 <- c $(0, \theta, 0,0,1)$

zbe $<-\operatorname{rep}(\theta$, times $=7)$

Any other constraint may be defined by modifying in a suitable way these three vectors and matrices Zga1, Zga2, Zbe. For instance, if we are interested in fixing the difficulty of (binary) item 4 to be equal to 2 (i.e., $\beta_{4}=2$ ), then we define $Z$ be and $z$ be as follows:

Zbe $<-\operatorname{diag}(7)[,-c(1,3,4,7)]$

Zbe

\begin{tabular}{|c|c|c|c|}
\hline \multicolumn{4}{|c|}{$[, 1][, 2][, 3]$} \\
\hline$[1]$, & 0 & 0 & \\
\hline$[2]$, & 1 & 0 & \\
\hline$[3]$, & 0 & 0 & \\
\hline$[4]$, & 0 & 0 & \\
\hline$[5]$, & 0 & 1 & \\
\hline$[6]$, & 0 & 0 & \\
\hline$[7]$, & 0 & 9 & \\
\hline
\end{tabular}

zbe $<-c(0,0,0,2,0,0,0)$

Function est_multi_poly_within supplies the following output:

- piv1 and piv2: vectors of the estimated weights of latent classes for $\boldsymbol{U}_{i}$ and $\boldsymbol{V}_{i}$, respectively; in the presence of individual covariates, these are averages of the individual-specific mass probabilities. 
- Th1 and Th2: matrices of estimated and constrained support points for each dimension (by row) and each latent class (by column) for $\boldsymbol{U}_{i}$ and $\boldsymbol{V}_{i}$, respectively.

- Bec: matrix of estimated and constrained item difficulty parameters; exact zeros correspond to identifiability constraints.

- ga1c and ga2c: vectors of estimated and constrained item discriminating parameters for $\boldsymbol{U}_{i}$ and $V_{i}$, respectively; exact ones correspond to identifiability constraints and NA to items that do not load on the latent variable.

- th1 $t$, th2 $t$, bet, ga1 $t$, and ga2t: estimated parameters (i.e., parameters without constraints) related to Th1, Th2, Bec, ga1c, ga2c, respectively.

- Th1s, Th2s, Becs, ga1cs, and ga2cs: standardized values of Th1, Th2, Bec, ga1c, and ga2c, respectively; in Th1s and Th2s classes are re-ordered according to the increasing values of the support points for the first dimension.

- piv1s and piv2s: the same as piv1 and piv2, but re-ordered according to Th1s and Th2s.

- fv1 and fv2: vectors indicating the reference items for each dimension of $\boldsymbol{U}_{i}$ and $\boldsymbol{V}_{i}$, respectively.

- Phi: conditional response probabilities for every item and each pair of latent classes of $\boldsymbol{U}_{i}$ and $V_{i}$.

- De1 and De2: matrices of estimated regression coefficients for the model on the class weights for $\boldsymbol{U}_{i}$ and $\boldsymbol{V}_{i}$, respectively, in the presence of individual covariates; for each covariate and the constant term, the number of estimated coefficients is equal to the number of latent classes minus one.

- Piv1, Piv2, Pp1, Pp2, and 1kv: optional output (obtained if output = TRUE) referred to the matrices of weights for every covariate configuration for latent variables $U_{i}$ and $V_{i}$, the matrices of the posterior probabilities for each response configuration and latent class for latent variables $U_{i}$ and $V_{i}$, and the values of the log-likelihood during the estimation process, respectively.

- XX1dis and XX2dis: design matrices for the covariates affecting the first and the second vector of latent variables, respectively (optional output obtained if output $=$ TRUE).

- $1 \mathrm{k}$ : value of the log-likelihood at convergence.

- np: number of estimated model parameters.

- aic, bic, and ent: AIC, BIC, and entropy indices, respectively.

- seDe1, seDe2, seTh1, seTh2, seBec, sega1, sega2, seth1 $t$, seth2t, sebet, sega1t, sega2t, and Vn: standard errors of the corresponding estimated parameters and estimated variance-covariance matrix (if out_se = TRUE).

Some relevant commands to display output from function est_multi_poly_within are based on the S3 methods summary for the main estimates; coef and confint for the point estimates and confidence intervals (at a specified level of confidence) of support points, item parameters, and regression coefficients; logLik for the value of log-likelihood at convergence; and vcov for the estimated variance-and-covariance matrix.

Another relevant function of package MLCIRTwithin is est_multi_poly_between, which performs the maximum likelihood estimation of an LC-IRT model under between-item multidimensionality. The main differences with respect to function est_multi_poly of the R package MultiLCIRT are that the latter does not allow for items with a different number of response categories and refers to a slightly different specification of item difficulties (for details see Bacci et al., 2014).

Input arguments required by est_multi_poly_between are very similar to those of function est_multi_poly_within. The main difference is that only one vector of latent variables is involved in the model specification. Consequently, the number of latent classes (input k) is common to all the dimensions and the multidimensional structure of the items is specified through one matrix (input multi), having one row for each dimension. Constraints on model parameters are also possible through a suitable definition of arguments Zth, zth, Zbe, zbe, Zga, and zga, whose functioning is the same as the corresponding arguments in function est_multi_poly_within. The function provides as its main output argument a vector of estimated average weights of the latent classes (output piv) and a matrix of estimated support points for each dimension and each latent class of the latent trait before (output Th) and after the standardization (output Ths). Besides, a matrix of difficulty item parameters (outputs Bec and, in the case of standardization, Becs), a vector of discriminating indices (output gac and, in the case of standardization, gacs), and a matrix of regression coefficients (output De) are provided, other than other output arguments similar to those above described for est_multi_poly_within, included the S3 methods.

Finally, we clarify that a model specification of type

out 1 <- est_multi_poly_between(S, $k=k 0, \quad l i n k=" g l o b a l ", \quad \operatorname{multi}=\operatorname{rbind}(1: 3,4: 6))$, 
with $\mathrm{k} \oslash$ latent classes, is substantially different from a model specification of type out2 <- est_multi_poly_within(S, k1 $=k 0, k 2=k 0, \quad$ link $=$ "global", multi1 $=c(1: 3)$,
multi2 $=c(4: 6))$.

In fact, the model corresponding to out 2 involves two completely independent latent variables, having incidentally the same number of latent classes: thus, an individual belonging to a specific class (say, class 1) according to the first latent variable may belong to any latent class under the second latent variable (say, class 2). On the contrary, model out 1 involves only one latent variable decomposed in two dimensions: thus, belonging to a given latent class under one dimension implies belonging to the same class under the other dimension. Overall, model out 2 has $k 0-1$ free parameters more than model out1.

\section{Functions search.model_within and search.model_between}

As outlined in Section "Likelihood inference," the selection of a two-tier LC-IRT model may be a quite demanding procedure, requiring the choice of the number of support points for the latent variables and a check for the possible presence of local maxima. Function search.model_within allows us to search for the global maximum of the log-likelihood of a model with a specific formulation (in terms of multidimensional structure, link function, and constraints on the item parameters) given a vector of possible number of latent classes to try for.

In practice, function search.model_wi thin applies function est_multi_poly_within a given number of times for each pair of values for $k_{1}$ and $k_{2}$, initializing the estimation algorithm with deterministic and random values of the model parameters and holding, for each pair of $k_{1}$ and $k_{2}$, that model with the highest value of the log-likelihood at convergence. To make the entire process computationally less demanding, the search of the global maximum may be performed with a relatively large tolerance level for checking convergence of the estimation algorithm. Then, in order to improve the precision of parameters estimates, the estimates provided by the model with the best value of the log-likelihood are used as starting values in the last step of the model selection process, using an augmented tolerance level. Note that when $k_{1}=1$ or $k_{2}=1$ the model estimation is actually performed by the function est_multi_poly_between, which is automatically retrieved by search.model_within.

The function at issue requires the following main input arguments:

- S, yv, X, link, disc, dif1, multi1, multi2, fort, disp, output, out_se, Zth1, zth1, Zth2, zth2, Zbe, zbe, Zga1, zga1, Zga2, and zga2: are the same as in function est_multi_poly_within.

- kv1 and kv2: vectors of number of latent classes to try for latent variable $\boldsymbol{U}_{i}$ and $\boldsymbol{V}_{i}$, respectively; single values are also allowed for.

- tol1 and tol2: tolerance levels (default value are $10^{-6}$ and $10^{-10}$, respectively) for checking convergence of the algorithm as relative difference between consecutive log-likelihoods. The value of tol 1 is used for checks based on deterministic and random starting values, whereas the value of tol2 is used for improving the precision of estimates for the model with the best log-likelihood level.

- nrep: constant value that drives the number of estimations of each model with random starting values, given by $\operatorname{nrep}\left(k_{1} k_{2}-1\right)$; the default value for nrep is 2 . In the case of nrep equal to 0 , only the estimation with deterministic starting values is performed.

Note that if single values for kv1 and kv2 are specified and nrep equals 0 , function search.model_wi thin performs just one call of function est_multi_poly_within (or function est_multi_poly_between if kv1 or kv2 equal 1) with option start = "deterministic".

Function search.model_within supplies the following output:

- aicv, bicv, and necv: vectors of AIC, BIC, and NEC indices, respectively, for each of the estimated models.

- errv: trace of any error occurred during the estimation process.

- $1 \mathrm{kv}$ : values of log-likelihood at convergence for each of the estimated models.

- out.single: output of each single model, similar to the output of est_multi_poly_within, with the addition of values of $k_{1}$ (output k1); $k_{2}$ (output k2); and the sequence of log-likelihoods (output $1 \mathrm{ktrace}$ ) for the deterministic start, for each random start, and for the final estimation provided by a tolerance level equal to tol2 (if tol2 $>$ tol1).

Finally, we outline that a function with input and output arguments similar to those of the function search.model_within is available to perform the model selection in the case of between-item multidimensional LC-IRT models. This function is named search.model_between and it relies on est_multi_poly_between. 


\section{Examples in $\mathbf{R}$}

In the following we illustrate package MLCIRTwithin through two data analysis examples. In Example 1 we describe the model selection procedure, as well as the interpretation of the output, considering a set of ordered items measuring two latent variables. In Example 2, the specification of constraints on the support points and on the item parameters is illustrated through the analysis of data concerning repeated item responses along two time occasions. The detailed software scripts to implement the two examples, named Example1.R and Example2.R, are available in the Supplementary Online Material at https://sites.google.com/site/bartstatistics/sm_mlcirtwithin.zip.

\section{Example 1: analysis of multidimensionality}

Data set SF12_nomiss, already provided in the R package MLCIRTwithin, refer to a sample of 493 oncological Italian patients who were asked to fill in the Italian validated Short Form 12 version 2 questionnaire (SF-12; Stewart and Ware, 1992; Ware et al., 2002) concerning the assessment of HQOL. The questionnaire is comprised by 12 items having five ordered response modalities, except items 2 and 3 having only three modalities; a high score means a worse level of HQOL and vice-versa (note that, in the original scoring system, modalities of items 9 and 10 are reversed). Also the age is available for each patient. In the following we show the first few records of the data set and the related summaries.

\section{library (MLCIRTwithin)}

data(SF12_nomiss)

head(SF12_nomiss)

$\begin{array}{rrrrrrrrrrrrrr} & Y 1 & Y 2 & Y 3 & Y 4 & Y 5 & Y 6 & Y 7 & Y 8 & Y 9 & Y 10 & Y 11 & Y 12 & \text { age } \\ 1 & 1 & 0 & 1 & 1 & 0 & 2 & 2 & 2 & 1 & 1 & 1 & 0 & 74.94593 \\ 2 & 0 & 0 & 1 & 1 & 2 & 1 & 2 & 1 & 0 & 2 & 1 & 1 & 84.49829 \\ 3 & 1 & 1 & 1 & 2 & 1 & 0 & 0 & 2 & 1 & 1 & 1 & 1 & 77.44285 \\ 4 & 3 & 2 & 2 & 4 & 4 & 4 & 4 & 3 & 4 & 4 & 4 & 4 & 80.55305 \\ 6 & 1 & 1 & 2 & 2 & 2 & 2 & 2 & 2 & 2 & 2 & 2 & 2 & 81.68104 \\ 7 & 1 & 0 & 1 & 3 & 2 & 2 & 1 & 2 & 1 & 2 & 1 & 3 & 78.55168\end{array}$

$\operatorname{str}$ (SF12_nomiss)

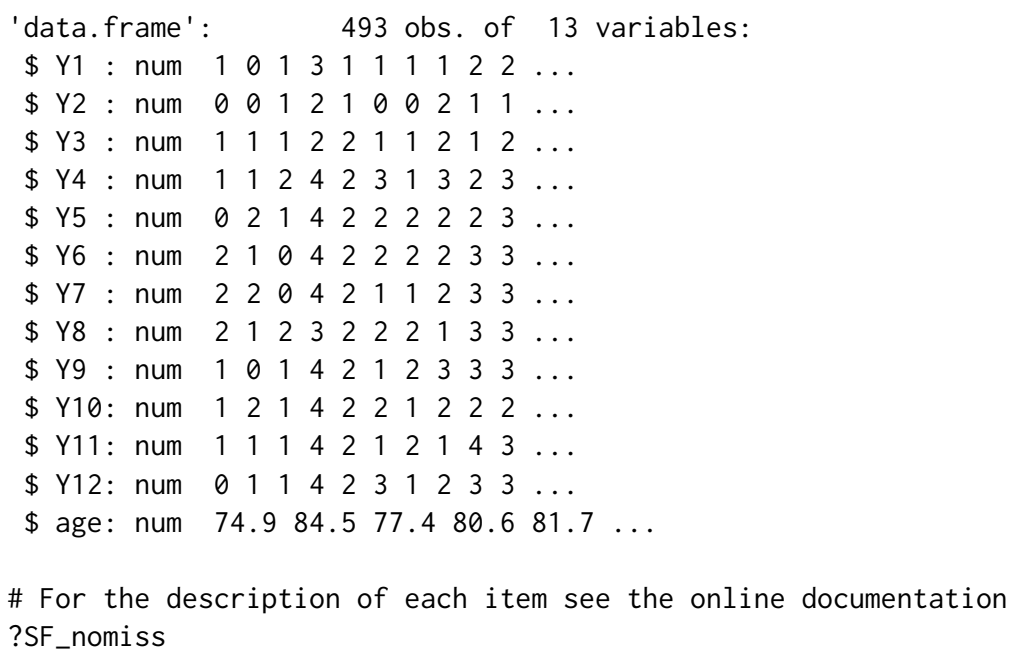

According to the main current literature (see, mainly, Ware et al., 2002), the SF-12 questionnaire may be used to properly evaluate two main aspects of HQOL: physical and emotional. The standard scoring algorithm for summarizing these two latent dimensions is based on an orthogonal factor analysis, on the basis of which positive and negative weights are assigned to each item. More in detail, items 1 to 5 and item 8 have positive weights for physical HQOL and negative weights for emotional HQOL, whereas items 6, 7, 9, 11, and 12 have negative weights for physical HQOL and positive weights for emotional HQOL; item 10 has positive weights for both components. According to this scoring system, the scores of physical and emotional HQOL result by a suitable weighted average of the item responses. 
A low score on physical HQOL has the following meaning (Ware and Gandek, 1998): substantial limitations in self-care, physical, social and role activities; severe bodily pain; frequent tiredness; health rated as poor. On the contrary, a high level of physical component corresponds to: no physical limitations, disabilities or decrements in well-being; high energy level; health rated as excellent. As regards the emotional component, a low score implies: frequent psychological distress, social and role disability due to emotional problems; health rated poor. On the other hand, a high level of emotional component corresponds to: frequent positive affect; absence of psychological distress and limitations in usual social activities due to emotional problems; health rated excellent.

The main drawback of the above algorithm based on the orthogonal factor analysis is that the summary score may be inconsistent due to weights of opposite sign for the same items, as higher emotional health scores drive physical health scores down and, similarly, higher physical health scores drive emotional health scores down (Farivar et al., 2007). An alternative approach for clustering patients according to their physical and emotional health status is based on IRT analysis (see, among others, Hays et al., 1993). In such a context, we analyze the multidimensional structure of SF-12 questionnaire through a two-dimensional model allowing items measuring both latent variables. In more detail, we compare several plausible multidimensional structures, defined through the following matrices, with the first one referred to the physical HQOL and the second one referred to the emotional HQOL:

Type 1: within-item multidimensional model with two independent latent variables and no shared item; items are allocated according to the sign of weights resulting by the factor analysis mentioned above:

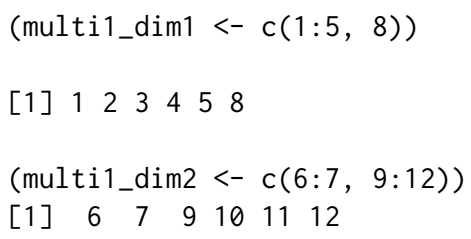

Type 2: model with two latent variables sharing items that do not explicitly affect a specific dimension

(multi2_dim1 <- c $(1: 5,8: 12))$

[1] $\begin{array}{llllllllll}1 & 2 & 3 & 4 & 5 & 8 & 9 & 10 & 11 & 12\end{array}$

(multi2_dim2 <- c(6:12, 1))

[1] $\begin{array}{llllllll}6 & 7 & 8 & 9 & 10 & 11 & 12 & 1\end{array}$

Type 3: multidimensional structure similar to the previous one, but with three items $(9,10$, and 11) assigned only to the emotional HQOL

(multi3_dim1 $<-c(1: 5,8,12))$

[1] $1 \begin{array}{lllllll}1 & 2 & 3 & 4 & 5 & 8 & 12\end{array}$

(multi3_dim2 <- $c(6: 12,1))$

[1] $\begin{array}{llllllll}6 & 7 & 8 & 9 & 10 & 11 & 12 & 1\end{array}$

Type 4: multidimensional structure similar to that defined through multi21 and multi22, but one more item (number 8), concerning the presence of pain, is assigned only to physical HQOL, since pain is usually intended in terms of physical health (i.e., bodily pain)

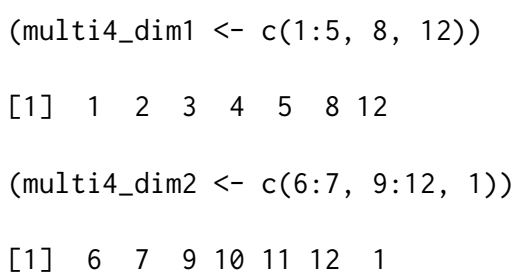

The allocation of every item according to one of the above proposed structures is suggested by the more or less explicit reference of the item text to physical or emotional component of HQOL (or to both of them).

Considering the possible multidimensional structures above defined, we focus on models with global logit link function and free discriminating item parameters; also the effect of age on the mass probabilities is investigated. 
\# Item responses and covariates

$\mathrm{S}<-$ SF12_nomiss [ , 1:12]

$X<-$ SF12_nomiss $[, 13]$

For each type of multidimensional structure, we select the optimal number of latent classes on the basis of BIC index according to the procedure described in Section "Likelihood inference". Also the check of local maxima solutions follows the same lines described therein. For these aims we use function search.model_within, as follows:

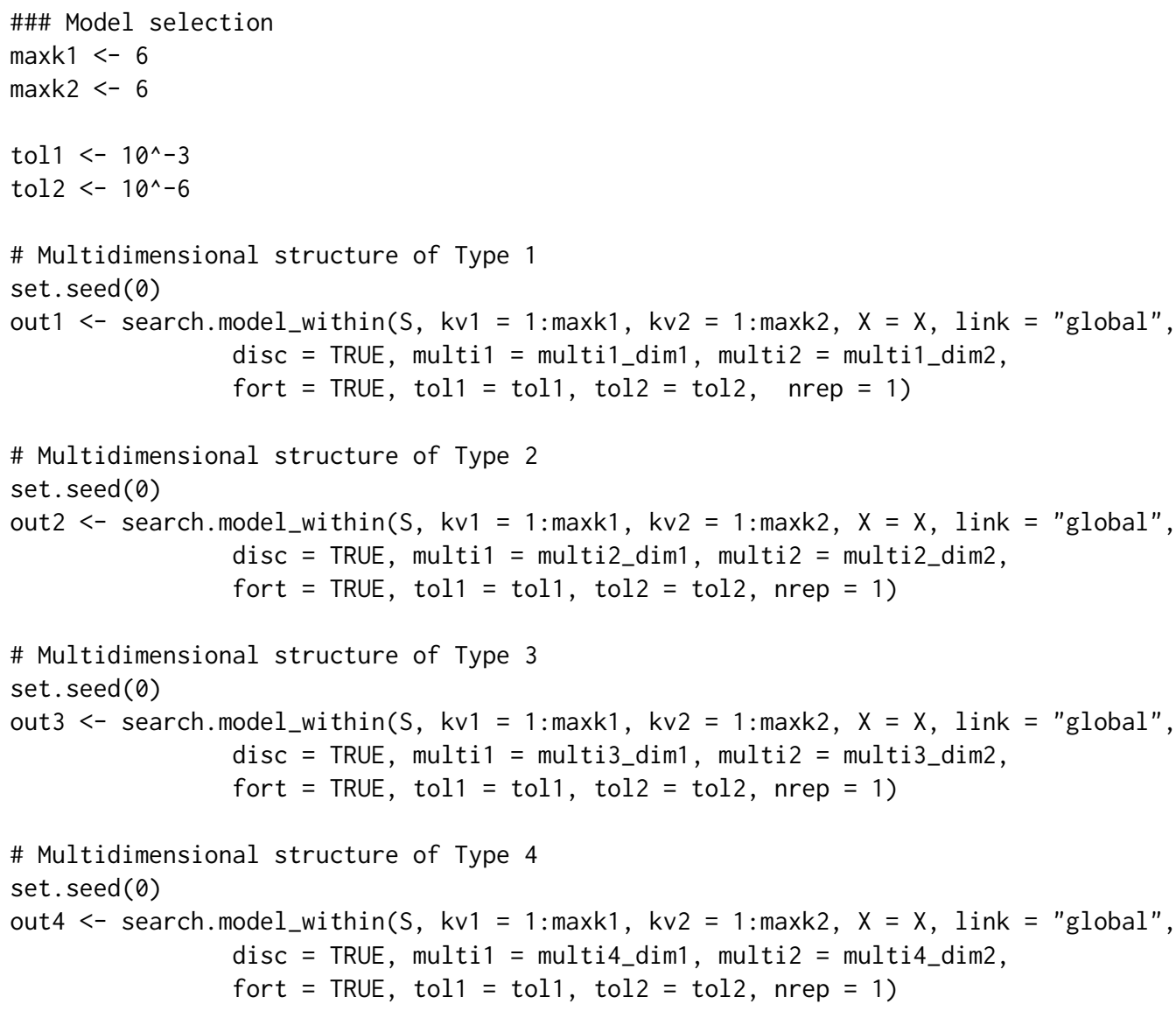

We advise that the entire estimation process may take a very long computational time; then, we suggest to reduce the tolerance level of the algorithm (we adopted $10^{-3}$ instead of the default value $10^{-6}$ for argument tol1 and $10^{-6}$ instead of the default value $10^{-10}$ for argument tol2) and the number of repetitions with random initializations (we specified nrep $=1$ instead of the default value nrep = 2). Outputs out1, out2, out3, and out4 are contained in the file 'Example1.RData', available in the supplementary online material at https://sites.google.com/site/bartstatistics/ sm_mlcirtwithin.zip.

In order to select the optimal model, values of BIC index are displayed in the following 36-by-4 matrix, having one row for each model and one column for each multidimensional structure:

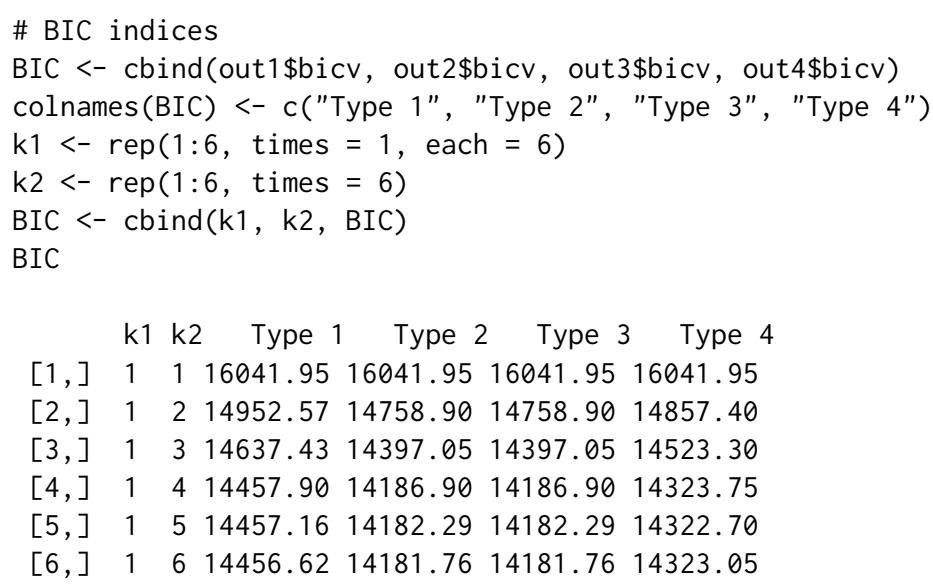

k1 k2 Type 1 Type 2 Type 3 Type 4

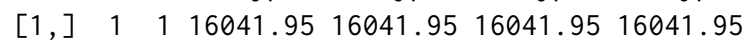

$[2,] \quad \begin{array}{lllllll}1 & 2 & 14952.57 & 14758.90 & 14758.90 & 14857.40\end{array}$

$[3] \quad 1 \quad 3 \quad 14637.43 \quad 14397.05 \quad 14397.05 \quad$,

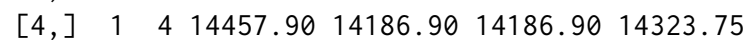

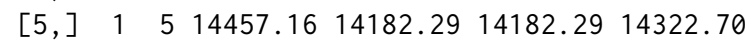

$[6,] \quad \begin{array}{lllllll}1 & 6 & 14456.62 & 14181.76 & 14181.76 & 14323.05\end{array}$ 


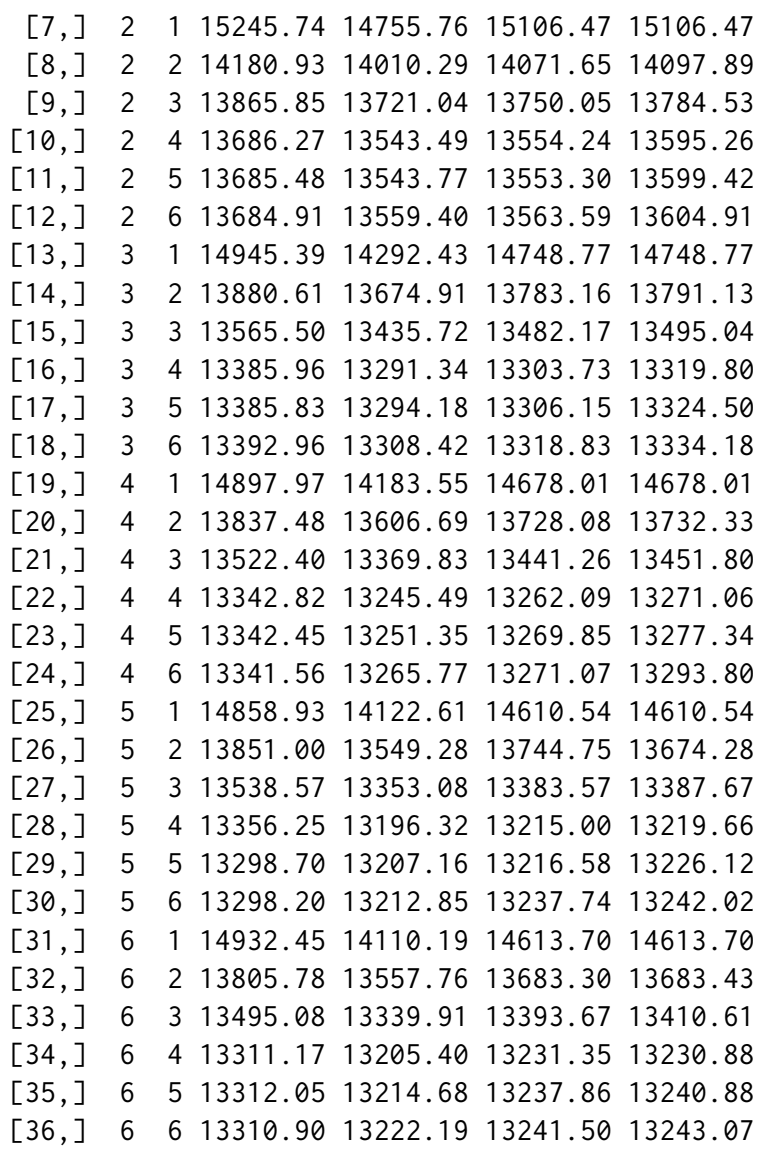

On the basis of the above matrix we observe that the minimum value of the BIC index, that is, 13196.32 , is displayed in column 2, denoting the multidimensional structure of type 2, and row 28, which refers to models with $k_{1}=5$ and $k_{2}=4$ latent classes. The output of the selected model is contained in the object out2\$out.single[[28]].

\# Minimum BIC

$\min (B I C[, 3: 6])$

[1] 13196.32

\# Detect model with the minimum BIC

$\operatorname{arrayInd}($ which.min(BIC $[,-c(1: 2)]), \cdot \operatorname{dim}=\operatorname{dim}(B I C))$

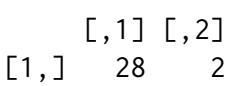

\# Number of support points for the best model out2\$out.single[[28]]\$k1

[1] 5

out2\$out.single[[28]]\$k2

[1] 4

\# Selected model

outsel = out $2 \$$ out.single[[28] $]$

We also observe that the same number of support points is selected for the other multidimensional structures with shared items (i.e., structures of types 3 and 4).

\# Minimum BIC and selected model for multidimensional structures of types 1, 3, 4 $\min (B I C[$, "Type 1"])

[1] 13298.2 
which.min(BIC[ ,"Type 1"])

[1] 30

$\min (B I C[$, "Type 3"])

[1] 13215

which.min(BIC[ ,"Type 3"])

[1] 28

$\min (B I C[$, "Type 4"])

[1] 13219.66

which.min(BIC[ ,"Type 4"])

[1] 28

After selecting the number of latent classes and the type of within-item multidimensional structure, we estimate again the selected model out with option out_se $=T$ for the computation of standard errors. To reduce the computational time, we use function est_multi_poly_within with option start = "external" and specified as input for options Phi, ga1c, ga2c, De1, and De2 the corresponding output from the selected model.

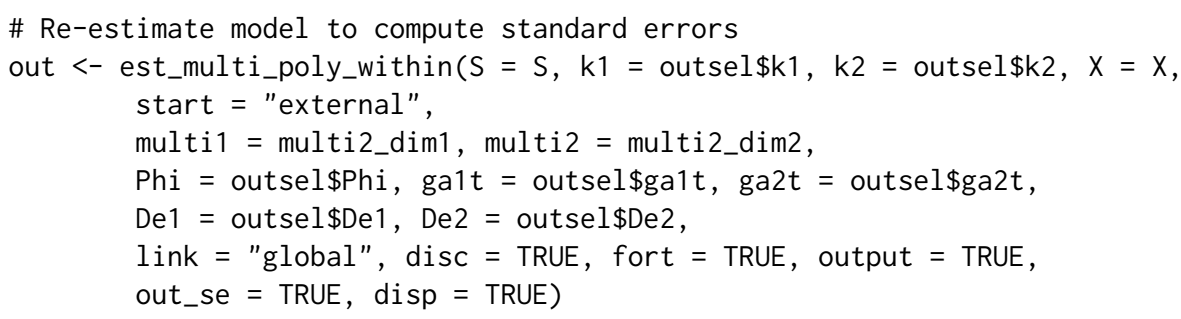

Details of the output of the estimated model may be displayed through the usual methods summary, coef, and confint.

\#\#\# Display output of the estimated model

\# summary (out)

\# coef(out)

\# confint(out)

\# Estimates of support points and average mass probabilities for physical HQOL

lv1 <- rbind(out\$Th1, t(out\$piv1))

rownames(lv1) <- c("Physical HQOL", "Average prob.")

round $(\operatorname{lv1}, 3)$

$\begin{array}{rrrrrr}1 & 2 & 3 & 4 & 5\end{array}$

$\begin{array}{llllll}\text { Physical HQOL } & 0.823 & 1.684 & 2.854 & 0.093 & -2.024\end{array}$

$\begin{array}{lllllll}\text { Average prob. } & 0.199 & 0.387 & 0.310 & 0.083 & 0.021\end{array}$

\# Estimates of support points and average mass probabilities for emotional HQOL

lv2 <- rbind(out\$Th2, t(out\$piv2))

rownames(lv2)<- c("Emotional HQOL", "Average prob.")

round (lv2, 3)

$\begin{array}{rrrrr}1 & 2 & 3 & 4\end{array}$

Emotional HQOL $0.471 \quad 11.526 \quad 7.5854 .151$

$\begin{array}{llllll}\text { Average prob. } & 0.149 & 0.141 & 0.390 & 0.321\end{array}$

According to the estimated model, patients are clustered in 5 latent classes denoting different levels of physical HQOL (output out\$Th1) and in 4 latent classes denoting different levels of emotional HQOL (output out\$Th2). To simplify the interpretation of the latent classes, it is useful to re-order and standardize the estimated support points. For this aim, function est_multi_poly_within provides the values of support points, which are standardized according to equations (14)-(18) and re-ordered 
according to increasing values of the first dimension of the corresponding latent variable (outputs out $\$$ Th1s and out $\$$ Th2s with related weights out $\$ p i v 1 s$ and out $\$ p i v 2 s$, respectively).

\# Standardized support points of physical HQOL

lv1s <- rbind(out\$Th1s, t(out\$piv1s))

rownames(lv1s) <- c("Stand. Physical HQOL", "Average prob.")

round $(\operatorname{lv} 1 \mathrm{~s}, 3)$

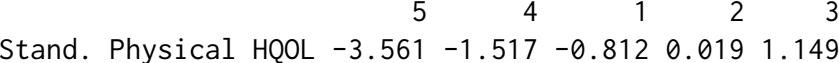

$\begin{array}{lllllll}\text { Average prob. } & 0.021 & 0.083 & 0.199 & 0.387 & 0.310\end{array}$

\# Standardized support points of emotional HQOL

lv2s <- rbind(out\$Th2s, t(out\$piv2s))

rownames(lv2s) <- c("Stand. Emotional HQOL", "Average prob.")

round $(\operatorname{lv} 2 s, 3)$

$\begin{array}{rrrr}1 & 4 & 3 & 2 \\ \text { Stand. Emotional HQOL }-1.667 & -0.553 & 0.486 & 1.679\end{array}$

$\begin{array}{lllll}\text { Average prob. } \quad 0.149 & 0.321 & 0.390 & 0.141\end{array}$

We observe that classes 5, 4, and 1 of physical HQOL and classes 1 and 4 of emotional HQOL collect patients with negative levels of the related latent trait, whereas patients with levels of HQOL above the mean belong to the remaining classes (i.e., classes 2 and 3 for physical HQOL and classes 3 and 2 for emotional HQOL). We also observe that the distribution of physical HQOL is strongly skewed to negative values, whereas that of emotional HQOL is symmetric (Figure 2).
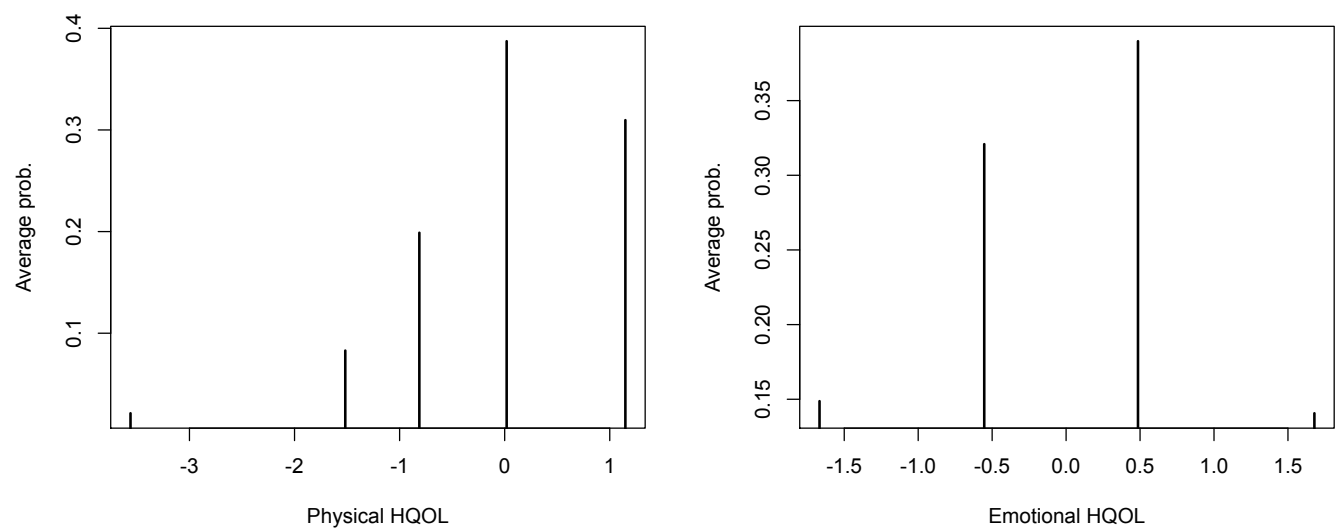

Figure 2: Standardized mass probability distribution of physical (left panel) and emotional (right panel) HQOL.

As concerns item parameters, the discriminating indices and the corresponding standard errors and confidence intervals are displayed as follows:

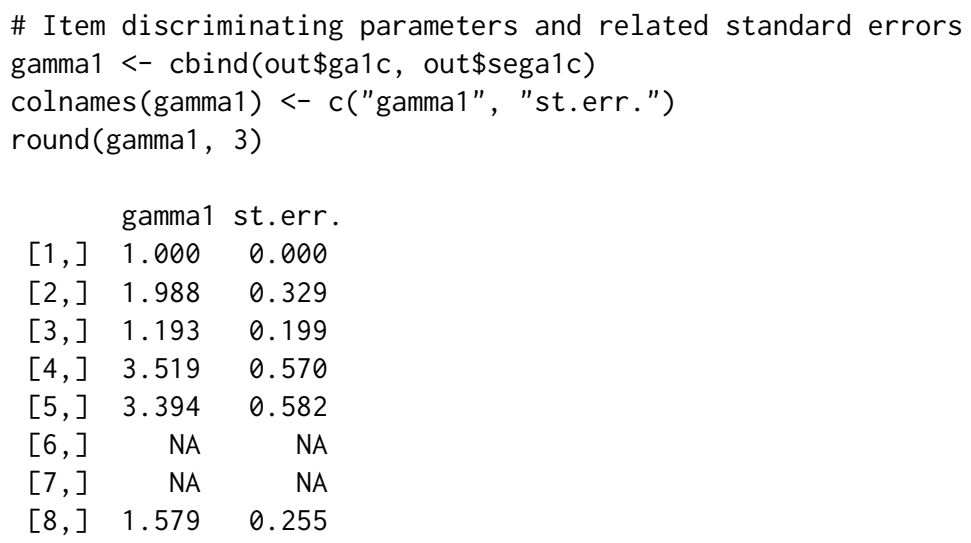




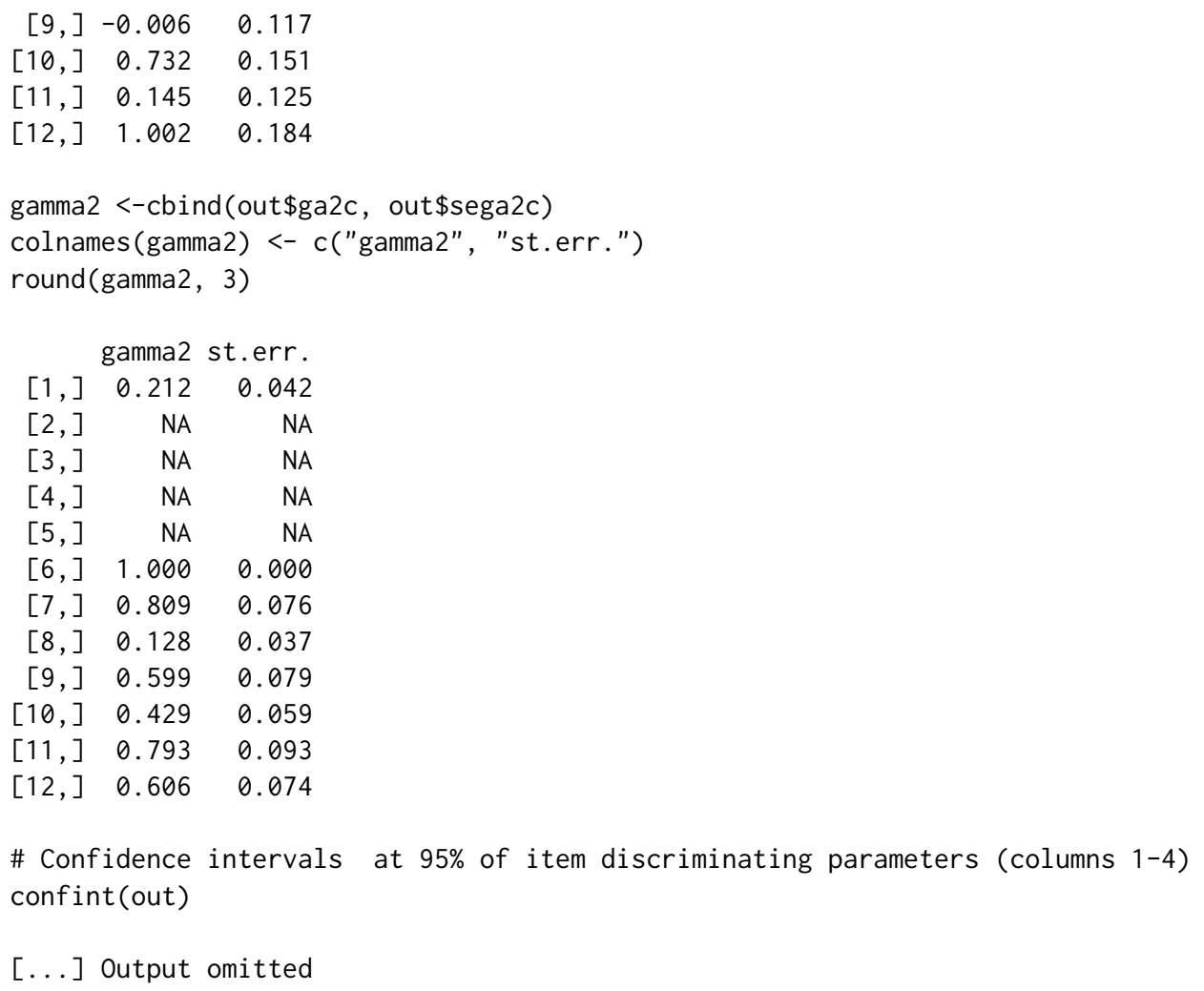

Confidence interval for the item parameters:

gamma1_1 gamma1_2 gamma2_1 gamma2_2 beta1_1 beta1_2 beta2_1 beta2_2 beta3_1 beta3_2 beta4_1 beta4_2

$\begin{array}{lllllllllllll}1.0000 & 1.0000 & 0.1293 & 0.2939 & 0.0000 & 0.0000 & 2.2927 & 3.0763 & 5.4703 & 6.6560 & 7.0976 & 8.9132\end{array}$

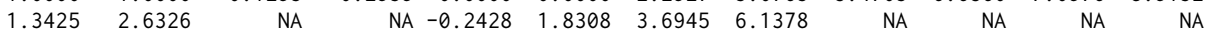

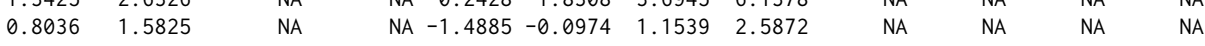

$\begin{array}{llllllllllll}2.4010 & 4.6368 & N A & N A & -1.3097 & 2.1941 & 1.2833 & 4.9066 & 5.2791 & 9.4358 & 8.5939 & 13.4278\end{array}$

$\begin{array}{llllllllllll}2.2542 & 4.5348 & N A & N A & -0.8427 & 2.5507 & 1.9437 & 5.5126 & 5.3510 & 9.4330 & 8.2434 & 12.8277\end{array}$

$\begin{array}{llllllllllll}\text { NA } & \text { NA } & 1.0000 & 1.0000 & 0.0000 & 0.0000 & 2.4866 & 3.9477 & 5.4657 & 7.6420 & 8.4771 & 11.2498 \\ \text { NA } & \text { NA } & 0.6612 & 0.9574 & -0.4397 & 0.7189 & 1.7730 & 3.0777 & 4.1602 & 5.8143 & 7.1608 & 9.3353\end{array}$

$\begin{array}{llllllllllll}1.0790 & 2.0796 & 0.0556 & 0.2010 & -2.8325 & -0.8067 & 0.3711 & 2.0693 & 3.0574 & 4.9355 & 4.5989 & 6.6225\end{array}$

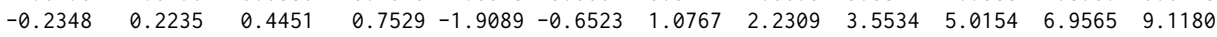

$\begin{array}{lllllllllllll}0.4366 & 1.0274 & 0.3135 & 0.5451 & -0.7823 & 0.3940 & 1.9615 & 3.1901 & 4.6207 & 6.1860 & 7.3341 & 9.3617\end{array}$

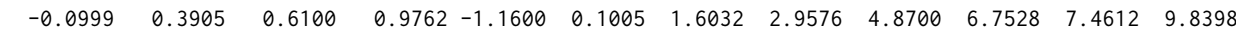

$\begin{array}{lllllllllllll}12 & 0.6411 & 1.3637 & 0.4611 & 0.7503 & -1.5644 & 0.0124 & 1.4481 & 2.8845 & 4.6337 & 6.4619 & 6.9167 & 9.0782\end{array}$

[...] Output omitted

In the previous output, the first two tables show the estimates of item discriminating parameters $\hat{\gamma}_{1 j}$ and $\hat{\gamma}_{2 j}$, respectively, and the related standard errors, whereas the last table shows the corresponding inferior and superior limits of the confidence intervals at $95 \%$ level. Entries denoted by NA refer to those items that do not load on the corresponding latent variable.

We observe that discriminating parameters are generally highly significant, with two notable exceptions: discriminating indices for items 9 and 11 referred to physical HQOL (i.e., parameters $\gamma_{19}$ and $\left.\gamma_{1,11}\right)$. This result suggests that these two items do not contribute in a significant way to the measurement of physical HQOL.

The standardized estimates of parameters $\hat{\gamma}_{1 j}^{*}$ and $\hat{\gamma}_{2 j}^{*}$ are provided by

\# Standardized discriminating parameters

gammas <- rbind(out\$ga1cs, out\$ga2cs)

rownames (gammas) <- c("Physical HQOL", "Emotional HQOL")

round (gammas, 3)

$[, 1] \quad[, 2] \quad[, 3] \quad[, 4] \quad[, 5] \quad[, 6] \quad[, 7] \quad[, 8] \quad[, 9] \quad[, 10][, 11][, 12]$ $\begin{array}{lllllllllllllllll}\text { Physical HQOL } & 1.036 & 2.059 & 1.236 & 3.645 & 3.516 & \text { NA } & \text { NA } & 1.636 & -0.006 & 0.758 & 0.151 & 1.038\end{array}$ $\begin{array}{llllllllllll}\text { Emotional HQOL } 0.699 & \text { NA NA NA NA } & 3.304 & 2.674 & 0.424 & 1.979 & 1.419 & 2.621 & 2.001\end{array}$

Note that the judgment about general health (item 1) is affected by both components of HQOL, but the physical dimension has a more relevant role $\left(\hat{\gamma}_{11}^{*}=1.036\right.$ vs $\left.\hat{\gamma}_{21}^{*}=0.699\right)$. On the contrary, the self-evaluation of the consequences of physical or emotional health on social activities (item 12) is mainly affected by the emotional component of HQOL $\left(\hat{\gamma}_{1,12}^{*}=1.038\right.$ vs $\left.\hat{\gamma}_{2,12}^{*}=2.001\right)$. 
We also highlight relevant differences among items in terms of difficulty, as results by the related standardized item parameters:

$$
\begin{aligned}
& \text { \# Standardized difficulty parameters } \\
& \text { round (out\$Becs, 3) } \\
& \text { cutoff } \\
& \begin{array}{lrrrr}
\text { item } & 1 & 2 & 3 & 4
\end{array} \\
& \begin{array}{lllll}
-2.929 & -0.245 & 3.134 & 5.076
\end{array}
\end{aligned}
$$

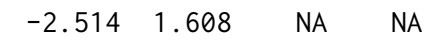

$$
\begin{aligned}
& \begin{array}{ll}
-2.778-0.115 \quad N A \quad N A
\end{array} \\
& \begin{array}{llll}
-5.414 & -2.761 & 1.501 & 5.155
\end{array} \\
& \begin{array}{lllll}
-4.795 & -1.921 & 1.743 & 4.887
\end{array} \\
& \begin{array}{lllll}
-5.979 & -2.762 & 0.575 & 3.884
\end{array} \\
& \begin{array}{llllll}
-4.700 & -2.414 & 0.148 & 3.409
\end{array} \\
& \begin{array}{lllll}
-5.215 & -2.175 & 0.601 & 2.215
\end{array} \\
& \begin{array}{llll}
-4.853 & -1.918 & 0.712 & 4.465
\end{array} \\
& 10-3.979-1.209 \quad 1.618 \quad 4.563 \\
& \begin{array}{llllll}
11 & -5.514 & -2.704 & 0.827 & 3.667
\end{array} \\
& \begin{array}{lllll}
12 & -6.066 & -3.124 & 0.258 & 2.707
\end{array}
\end{aligned}
$$

In particular, positive responses to items related only (items 4 and 5) or mainly (item 1) to physical HQOL require a level of the latent trait in average higher than items related only (items 6 and 7) or mainly (items 9, 11, and 12) to emotional HQOL. In other words, the emotional component of HQOL interferes less than physical component of HQOL on work, daily activities, and social life.

In the estimated model we assume an effect of patient's age on the HQOL, according to the multinomial logit parametrization, as in equation (3). Estimates of regression coefficients $\delta_{1 h_{1}}$ and $\delta_{2 h_{2}}$, with $h_{1}=2,3,4,5$ and $h_{2}=2,3,4$, and related standard errors, are provided by:

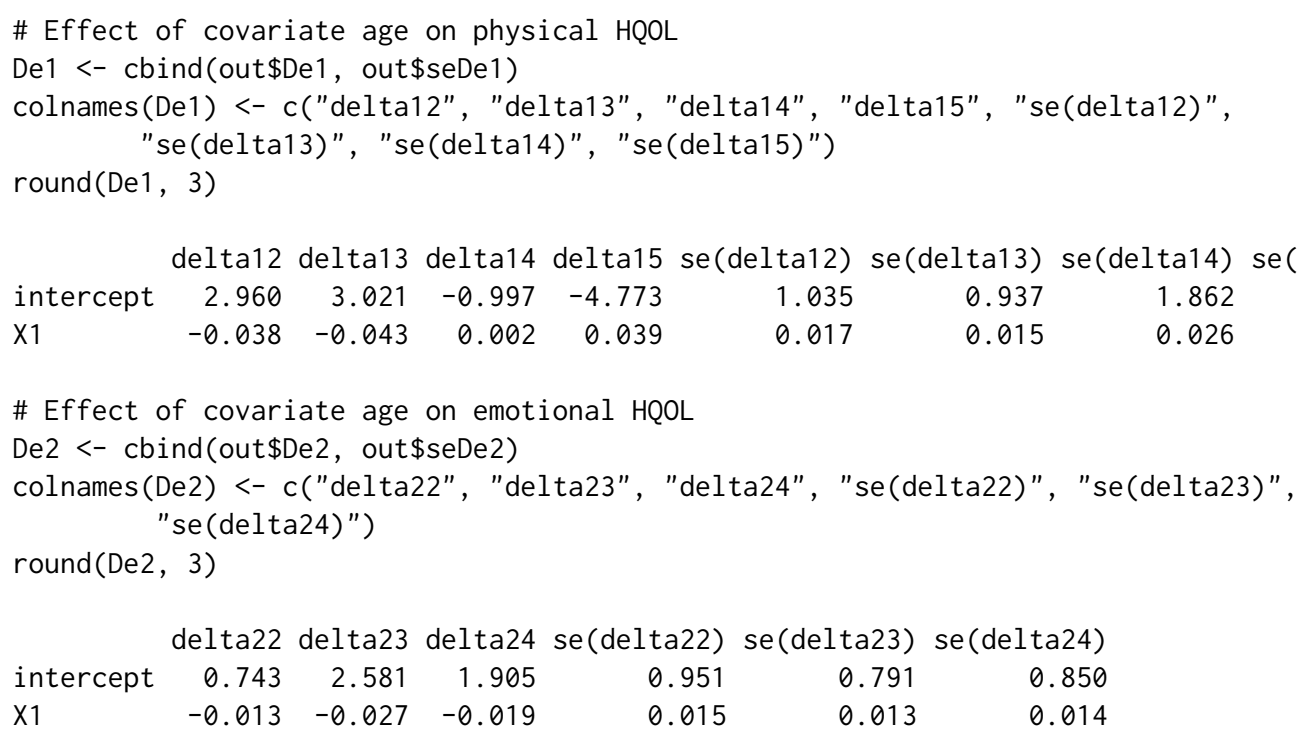

\# Confidence intervals at $95 \%$ of regression coeffic. for physical and emotional HQOL confint (out)

$[\ldots]$ Output omitted

Confidence interval for the regression coefficients for the 1st latent variable: logit

$$
\text { 2_1 2_2 3_1 3_2 } 4 \_1 \quad 4 \_2 \quad 5 \_1 \quad 5 \_2
$$

$\begin{array}{lllllllll}\text { intercept } & 0.9301 & 4.9890 & 1.1833 & 4.8581 & -4.6451 & 2.6520 & -9.5808 & 0.0355\end{array}$

$\begin{array}{llllllllll}\mathrm{X} 1 & -0.0709 & -0.0055 & -0.0717 & -0.0146 & -0.0489 & 0.0528 & -0.0295 & 0.1075\end{array}$

Confidence interval for the regression coefficients for the 2nd latent variable: logit

$$
\text { 2_1 2_2 } 3 \_1 \quad 3 \_2 \quad 4 \_1 \quad 4 \_2
$$

$\begin{array}{lllllll}\text { intercept }-1.1204 & 2.606 & 1.0306 & 4.1313 & 0.2383 & 3.5707\end{array}$

$\begin{array}{llllllll}\mathrm{x} 1 & -0.0434 & 0.017 & -0.0521 & -0.0021 & -0.0458 & 0.0081\end{array}$ 
We observe a negative, although not particularly significant, effect of age on the probability to belong to a specific latent class with respect to the worst one (i.e., class 1), for both components of HQOL. A more parsimonious and more easily interpretable solution is provided by specifying a global logit parametrization through option glob = TRUE of the function est_multi_poly_within, as follows:

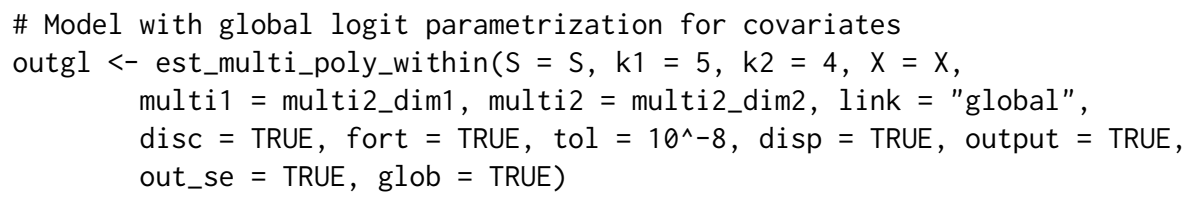

\# Confidence intervals at $95 \%$ of regression coeffic. for physical and emotional HQOL confint (outgl)

[...] Output omitted

Confidence interval for the regression coefficients for the 1 st latent variable:

$\operatorname{logit}$

$\begin{array}{lrr} & -1 & -2 \\ \text { cutoff1 } & 4.0265 & 6.1586 \\ \text { cutoff2 } & 1.9112 & 3.7427 \\ \text { cutoff3 } & 0.1816 & 1.9187 \\ \text { cutoff4 } & -1.7110 & 0.1575 \\ \text { X1 } & -0.0422 & -0.0132\end{array}$

Confidence interval for the regression coefficients for the 2nd latent variable: logit

$\begin{array}{lrr} & -1 & -2 \\ \text { cutoff1 } & 1.3987 & 3.2124 \\ \text { cutoff2 } & -0.2229 & 1.5509 \\ \text { cutoff3 } & -2.2153 & -0.4088 \\ \text { X1 } & -0.0240 & 0.0049\end{array}$

In such a case just one regression coefficient for each latent variable is estimated instead of $k_{1}-1$ or $k_{2}-1$, denoting a negative effect of patient's age on the logit to belong to a specific class or higher with respect to a lower class. 


\section{Example 2: analysis of repeated item responses}

An interesting example of a real problem that can be solved through the discrete two-tier models is encountered when a questionnaire is used on multiple waves to measure the same latent variable at each time occasion. In such a situation, following Cai (2010) we distinguish a "specific" dimension $U_{i d_{1}}$ for each item within the questionnaire to capture the dependence between an individual's responses to the same item $d_{1}\left(d_{1}=1, \ldots, D_{1}, D_{1}=r\right)$ at the different occasions, and we introduce one "primary" dimension $V_{i d_{2}}$ for each wave $d_{2}\left(d_{2}=1, \ldots, D_{2}\right)$, representing the latent variable of interest at a given occasion. Through a suitable choice of identifiability and equality constraints on the item parameters, it is possible to estimate the support points of $V_{i}$ and, then, obtain a class-specific time trajectory of the latent variable measured by the questionnaire. The resulting multidimensional structure is illustrated in Figure 3 for the case $D_{2}=2$.

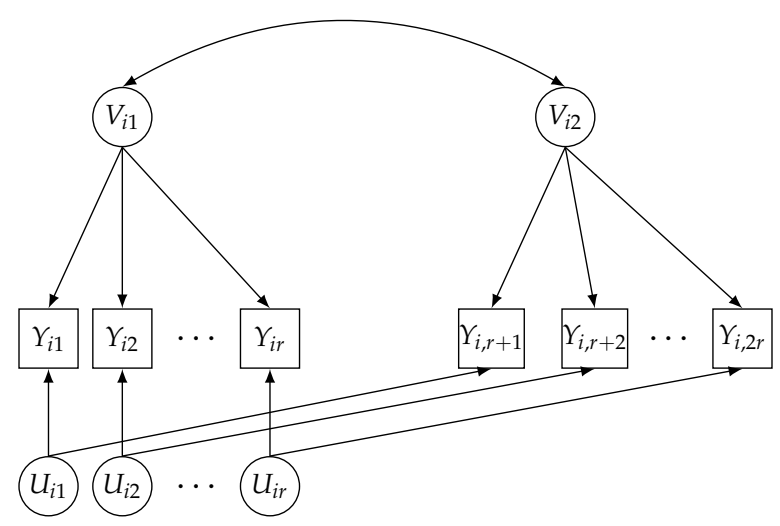

Figure 3: Path diagram of the discrete two-tier model for longitudinal data for $r$ items and $D_{2}=2$ waves.

To illustrate specification and estimation of the discrete two-tier model for longitudinal data we refer to a simulated data set about 10 different types of crime committed by a cohort of 10,000 hypothetical subjects on 6 waves; each crime corresponds to a binary item, which assumes value 1 if the crime was committed and 0 otherwise. The latent variable of interest denotes the propensity to commit a crime at each time occasion. The data set is available from the R package LMest (Bartolucci and Pandolfi, 2016).

library(MLCIRTwithin)

\begin{tabular}{|c|c|c|c|c|c|c|c|c|c|c|c|c|c|}
\hline \multicolumn{14}{|c|}{$\begin{array}{l}\text { \#\#\# Load and prepare data } \\
\text { library(LMest) } \\
\text { data(data_criminal_sim) } \\
\text { data_criminal_sim[1:12,] }\end{array}$} \\
\hline & id & ex & time & $\mathrm{y} 1$ & y2 & y3 & $\mathrm{y} 4$ & $y 5$ & $y 6$ & y7 & y8 & y9 & 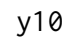 \\
\hline$[1]$, & 1 & 1 & 1 & 0 & 0 & 0 & 0 & 0 & 0 & 0 & 0 & 0 & 0 \\
\hline [2, & 1 & 1 & 2 & 0 & 0 & 0 & 0 & 0 & 0 & 0 & 0 & 0 & \\
\hline 3,$]$ & 1 & 1 & 3 & 0 & 0 & 0 & 0 & 0 & 0 & 0 & 0 & 0 & 0 \\
\hline$[4]$, & 1 & 1 & 4 & 0 & 0 & 0 & 0 & 0 & 0 & 0 & 0 & 0 & 0 \\
\hline 5,$]$ & 1 & 1 & 5 & 0 & 0 & 0 & 0 & 0 & 0 & 0 & 0 & 0 & 0 \\
\hline$[6]$. & 1 & 1 & 6 & 0 & 0 & 0 & 0 & 0 & 0 & 0 & 0 & 0 & 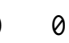 \\
\hline$[7]$, & 2 & 1 & 1 & 0 & 0 & 0 & 0 & 0 & 0 & 0 & 0 & 0 & 0 \\
\hline$[8]$, & 2 & 1 & 2 & 0 & 0 & 0 & 0 & 0 & 0 & 0 & 0 & 0 & 0 \\
\hline$[9]$, & 2 & 1 & 3 & 0 & 0 & 0 & 0 & 0 & 0 & 0 & 0 & 0 & 0 \\
\hline a 7 & 2 & 1 & 4 & 0 & 0 & 0 & 0 & 0 & 0 & 0 & 0 & 0 & 0 \\
\hline & 2 & 1 & 5 & 0 & 0 & 0 & 0 & 0 & 0 & 0 & 0 & 0 & 0 \\
\hline 2, & 2 & 1 & 6 & 0 & 0 & 0 & 0 & 0 & 0 & 0 & 0 & 0 & \\
\hline
\end{tabular}

In order to simplify the illustration of the application, we keep the first two waves and five types of crime, as follows:

\# Keep items: y1,y3,y5,y7,y10; keep occasions: 1, 2

criminal_red <- data_criminal_sim[(data_criminal_sim[,3]==1 | data_criminal_sim[,3]==2), $c(1: 3,4,6,8,10,13)]$ 
Moreover, in order to estimate the model at issue through the R package MLCIRTwithin, we need to reshape the data from "long" to "wide" format; we also aggregate records corresponding to the same patterns. Note that, after reshaping the data set, the total amount of items is 10 , that is, 5 items observed at time $1(j=1, \ldots, 5)$ and the same 5 items observed again at time $2(j=6, \ldots, 10)$.

\# Data reshape in wide format

criminal_red <- data.frame(criminal_red)

crim_wide <- reshape(criminal_red, v.names = c("y1", "y3", "y5", "y7", "y10"), timevar = "time", idvar = "id", direction = "wide")

$\operatorname{head}($ crim_wide)

id sex y1.1 y3.1 y5.1 y7.1 y10.1 y1.2 y3.2 y5.2 y7.2 y10.2

$\begin{array}{lllllllllllll}1 & 1 & 1 & 0 & 0 & 0 & 0 & 0 & 0 & 0 & 0 & 0 & 0 \\ 3 & 2 & 1 & 0 & 0 & 0 & 0 & 0 & 0 & 0 & 0 & 0 & 0 \\ 5 & 3 & 1 & 0 & 0 & 0 & 0 & 0 & 0 & 0 & 0 & 0 & 0 \\ 7 & 4 & 1 & 0 & 0 & 0 & 0 & 0 & 0 & 0 & 0 & 0 & 0 \\ 9 & 5 & 1 & 0 & 0 & 0 & 0 & 0 & 0 & 0 & 0 & 0 & 0 \\ 11 & 6 & 1 & 0 & 0 & 0 & 0 & 0 & 0 & 0 & 0 & 0 & 0\end{array}$

dim(crim_wide)

[1] $10000 \quad 12$

\# Aggregate records with the same pattern

crim_wide <- as.matrix(crim_wide)

crim_wide2 <- aggr_data(crim_wide[, -1])

\# Item responses, covariates, and vector of weights

$\mathrm{S}<-$ crim_wide $2 \$$ data_dis $[,-1]$

$X<-$ crim_wide $2 \$$ data_dis $[, 1] ; X<-X-1$

yv $<-$ crim_wide2\$freq

The multidimensional structure is defined through the specification of matrices multi1 and multi2, with multi 1 referring to specific dimensions capturing the dependence between responses to the same item at the two different waves, whereas mult $i 2$ refers to the propensity to commit a crime at time 1 and at time 2. Then, $U_{i}=\left(U_{i 1}, \ldots, U_{i 5}\right)^{\prime}$ and $V_{i}=\left(V_{i 1}, V_{i 2}\right)^{\prime}$; we also assume $k_{1}=k_{2}=2$.

\#\#\# Define the multidimensional structure

multi1 <- $\operatorname{matrix}(\theta$, nrow=5, ncol=2)

multi2 <- $\operatorname{matrix}(0$, nrow $=2$, ncol=5)

multi1 $[1]<,-c(6,1)$

multi1 $[2]<,-c(2,7)$

multi1 $[3]<,-c(3,8)$

multi1 $[4]<,-c(4,9)$

multi1 $[5]<,-c(5,10)$

multi2[1,]<-c(1:5)

multi2[2,] $<-c(7,6,8: 10)$

multi1

\begin{tabular}{|c|c|c|c|}
\hline \multicolumn{4}{|c|}{$[, 1][, 2]$} \\
\hline$[1]$, & 6 & 1 & \\
\hline$[2]$, & 2 & 7 & \\
\hline$[3]$, & 3 & 8 & \\
\hline$[4]$, & 4 & 9 & \\
\hline $\begin{array}{l}{[5,]} \\
\text { multi2 }\end{array}$ & 5 & 10 & \\
\hline & 1] & {$[-2][, 3]$} & {$[, 4][, 5]$} \\
\hline,$]$ & 1 & 23 & 4 \\
\hline , ] & 7 & 6 & $9 \quad 10$ \\
\hline
\end{tabular}

\# Number of latent classes

$\mathrm{k} 1<-2$

$k 2<-2$

The next step of the model specification consists in fixing some support points to identify the model and adding equality constraints on difficulties and discriminating indices to properly account for the longitudinal data structure, similarly to Cai (2010). In more detail, we fix the support points of the latent variable $\boldsymbol{U}_{i}$, that is, $\boldsymbol{u}=(-1,-1,-1,-1,-1,1,1,1,1,1)^{\prime}$, and on the first dimension of the latent variable $V_{i}$, whereas the support points on the second dimension of $V_{i}$ are freely estimated, that is, 
$v=\left(-1, v_{12}, 1, v_{22}\right)^{\prime}$ : in such a way, we estimate how the propensity to commit a crime changes over the time. Moreover, we constrain difficulty and discriminating parameters of each item at time 1 to be equal to the parameters of the same item at time 2 , that is, $\beta_{j}=\beta_{5+j}, \gamma_{1 j}=\gamma_{1,5+j}, \gamma_{2 j}=\gamma_{2,5+j}$, with $j=1, \ldots, 5$.

\#\#\# Specification of model constraints

\# Fix support points on latent variable $U$

\# Fix support points on the first dimension of latent variable $V$

\# Free support points on the second dimension of latent variable $V$

$($ Zth1 $<-\operatorname{matrix}(\theta, \operatorname{nrow}(\operatorname{multi1}) * \mathrm{k} 1, \theta))$

$[1$,

$[2$,

$[3$,

$[4$,

$[5$,

$[6$,

$[7$,

$[8$,

$[9$,

$[10$,

$(z$ th1 $<-c(\operatorname{rep}(-1$, times $=\operatorname{nrow}(\operatorname{multi} 1)), \quad \operatorname{rep}(1, \operatorname{times}=\operatorname{nrow}(\operatorname{multi1}))))$

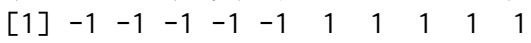

$($ Zth2 $<-\operatorname{diag}(\operatorname{nrow}(\operatorname{multi2}) * k 2)[,-c(1,3)])$

$[1],[, 1][, 2]$

$[2$,

$[2] \quad 1 \quad$,

$[4] \quad 0 \quad$,

(zth2 <- c(-1, $0,1,0))$

[1] $-1 \quad 0 \quad 1 \quad 0$

\# Equality constraints on difficulties and discriminating indices to account

\# for the longitudinal data structure

(Zbe <- matrix(1, nrow(multi2), 1) \%x\% diag(nrow(multi1)))

$$
[, 1][, 2][, 3][, 4][, 5]
$$

$\begin{array}{cccccc}{[1,]} & 1 & 0 & 0 & 0 & 0 \\ {[2,]} & 0 & 1 & 0 & 0 & 0 \\ {[3,]} & 0 & 0 & 1 & 0 & 0 \\ {[4,]} & 0 & 0 & 0 & 1 & 0 \\ {[5,]} & 0 & 0 & 0 & 0 & 1 \\ {[6,]} & 1 & 0 & 0 & 0 & 0 \\ {[7,]} & 0 & 1 & 0 & 0 & 0 \\ {[8,]} & 0 & 0 & 1 & 0 & 0 \\ {[9,]} & 0 & 0 & 0 & 1 & 0 \\ {[10,]} & 0 & 0 & 0 & 0 & 1 \\ \text { Zga2 <- Zbe } & & & & \\ \text { Zga1 <- Zbe } & & & & \end{array}$

The final step consists in estimating the model according to the structure specified above. The main difference with respect to the model presented in the previous section (Example 1) is that now all items are shared by both the latent variables $\boldsymbol{U}_{i}$ and $\boldsymbol{V}_{i}$ and suitable constraints on the model parameters are introduced through arguments Zth1, zth1, Zth2, zth2, Zbe, Zga1, and Zga2, according to equations (7)-(11).

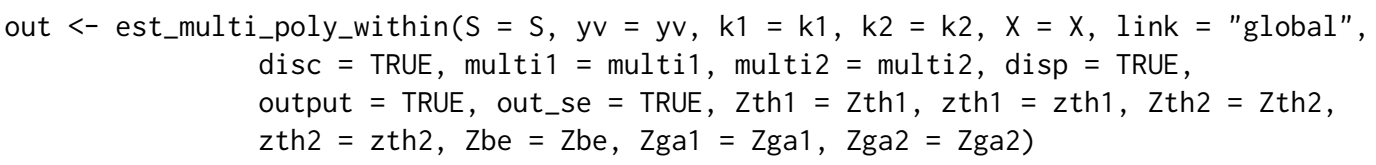

Estimates of support points and item parameters may be displayed through the usual methods 
summary, coef, and confint:

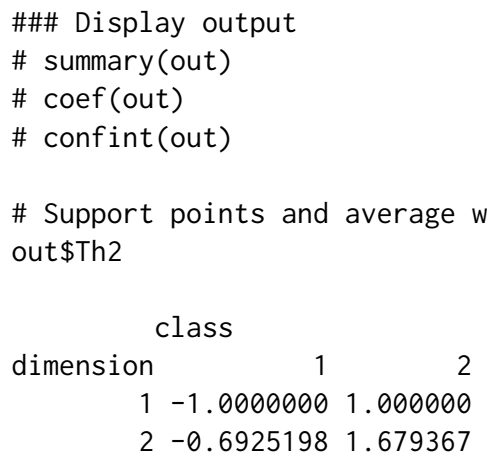

out\$piv2

$[, 1]$

$[1] \quad$,

$[2] \quad$,

\# Conditional response probabilities for latent variable $\mathrm{V}$

\# Note that the latent classes are numbered following an increasing order,

\# starting from latent variable $U$

round(out\$Phi[, , 3:4], 3)

, class $=3$

\begin{tabular}{lllllllllll}
\multicolumn{2}{c}{ item } \\
category & 1 & 2 & 3 & 4 & 5 & 6 & 7 & 8 & 9 & 10
\end{tabular}

$\begin{array}{lllllllllllll}0 & 0.994 & 0.982 & 0.944 & 0.986 & 0.998 & 0.991 & 0.974 & 0.919 & 0.981 & 0.997\end{array}$

$\begin{array}{lllllllllllll}1 & 0.006 & 0.018 & 0.056 & 0.014 & 0.002 & 0.009 & 0.026 & 0.081 & 0.019 & 0.003\end{array}$

, class $=4$

\begin{tabular}{rrrrrrrrrrr}
\multicolumn{2}{l}{ item } & & & & & & \\
category & 1 & 2 & 3 & 4 & 5 & 6 & 7 & 8 & 9 & 10 \\
0 & 0.897 & 0.799 & 0.551 & 0.874 & 0.882 & 0.758 & 0.618 & 0.335 & 0.758 & 0.628 \\
1 & 0.103 & 0.201 & 0.449 & 0.126 & 0.118 & 0.242 & 0.382 & 0.665 & 0.242 & 0.372
\end{tabular}

We observe that $91.8 \%$ of the individuals belong to class 1 , which is characterized by the smallest propensity to commit a crime, whereas the remaining $8.24 \%$ of individuals are allocated in class 2 . Both classes present a tendency to increase the propensity to commit a crime from time 1 to time 2 . Indeed, the estimated support points of the propensity to commit a crime at time 2 (i.e., estimates of $v_{12}$ and $\left.v_{22}\right)$ are higher than the corresponding values at time 1 , for both the latent classes $(-0.693$ vs -1 for class 1 and 1.679 vs 1 for class 2). Moreover, the conditional probabilities of observing a given crime is higher for items observed at time 2, that is, items 6 to 10 , with respect to the same items observed at time 1 , that is, items 1 to 5 , mainly in the case of latent class 2 . For instance, for an individual belonging to class 2 the conditional probability of observing a crime of type 1 equals $10.3 \%$ at time 1 and it increases to $24.2 \%$ at time 2 .

Finally, we outline that the constraints specified through matrices Zbe, Zga1, and Zga2 result in the following estimates, which are equal for every pair of items referring to the same type of crime (i.e., items 1-6, 2-7, 3-8, 4-9, 5-10):

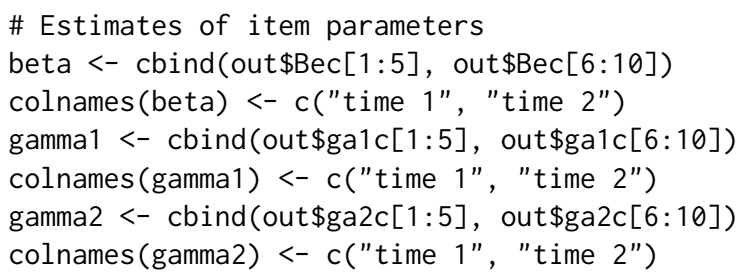

beta 
$[2] \quad$,

$[3]$,

$[4] 5.991825 \quad$,

$[5] 5.544927 \quad$,

gamma1

time 1 time 2

$[1] \quad$,

$[2] \quad 2.195804 \quad$,

$[3] 1.184268 \quad$,

$[4]$,

$[5]$,

gamma2

time 1 time 2

$[1]$,

$[2] \quad 1.317424 \quad$,

$[3] \quad$,

$[4] \quad 1.169848 \quad$,

$[5]$,

\section{Summary}

In this paper we illustrate the R package MLCIRTwithin, whose main function est_multi_poly_wi thin implements an Expectation Maximization based approach to estimate the parameters of two-tier latent class item response theory (IRT) models. This class of models, which extends in a flexible way the class of basic IRT models, is based on two independent vectors of latent variables. Moreover, two main assumptions hold: (i) items are allowed to measure one or two latent variables (within-item multidimensionality) and (ii) latent variables are assumed to have a discrete distribution with a finite number of support points, which identify homogeneous latent classes of individuals, and related mass probabilities that may depend on individual covariates.

We illustrate the R package through two examples based on data about the measurement of healthrelated quality of life in cancer patients (Example 1) and about the measurement of the propensity to commit a crime in two time occasions (Example 2). The first example investigates the multidimensional structure of the questionnaire and is focused on the interpretation of the estimated model parameters. The second example illustrates how to treat longitudinal item responses through the specification of suitable constraints on support points and item parameters.

\section{Acknowledgements}

Both authors acknowledge the financial support from the grant FIRB ("Futuro in ricerca") 2012 on "Mixture and latent variable models for causal inference and analysis of socio-economic data", which is funded by the Italian Government (RBFR12SHVV). Authors are also grateful to Dr. G. Miccinesi of the Istituto per lo Studio e la Prevenzione Oncologica (Florence, IT) for making available the data used in Example 1.

\section{Bibliography}

R. J. Adams, M. Wilson, and W.-C. Wang. The multidimensional random coefficients multinomial logit. Applied Psychological Measurement, 21:1-23, 1997. [p139]

A. Agresti. Categorical Data Analysis. Wiley, Hoboken, NJ, 2013. [p141]

H. Akaike. Information theory and an extension of the maximum likelihood principle. In B. N. Petrov and F. Csaki, editors, Second International Symposium of Information Theory, pages 267-281, Budapest, 1973. Akademiai Kiado. [p143]

D. Andrich. A rating formulation for ordered response categories. Psychometrika, 43:561-573, 1978. [p141]

S. Bacci and F. Bartolucci. A multidimensional finite mixture structural equation model for nonignorable missing responses to test items. Structural Equation Modeling: A Multidisciplinary Journal, 22: 352-365, 2015. [p139, 143] 
S. Bacci, F. Bartolucci, and M. Gnaldi. A class of multidimensional latent class IRT models for ordinal polytomous item responses. Communications in Statistics - Theory and Methods, 43:787-800, 2014. [p139, 141, 148]

F. Bartolucci. A class of multidimensional IRT models for testing unidimensionality and clustering items. Psychometrika, 72:141-157, 2007. [p139]

F. Bartolucci and S. Bacci. MLCIRTwithin: Latent Class Item Response Theory (LC-IRT) Models under Within-Item Multidimensionality. R package version 2.1, URL https://cran.rproject.org/web/packages/MLCIRTwithin, 2016. [p139, 144]

F. Bartolucci and S. Pandolfi. LMest: Latent Markov Models with and without Covariates. R package version 2.2-0, URL https:/ / cran.r-project.org/web/packages/LMest, 2016. [p159]

F. Bartolucci, S. Bacci, and M. Gnaldi. MultiLCIRT: An R package for multidimensional latent class item response models. Computational Statistics \& Data Analysis, 71:971-985, 2014. [p144]

F. Bartolucci, S. Bacci, and M. Gnaldi. Statistical Analysis of Questionnaires: a Unified Approach based on R and Stata. Chapman \& Hall, CRC Press, Boca Raton, FL, 2015. [p139, 141, 144]

F. Bartolucci, S. Bacci, and M. Gnaldi. MultiLCIRT: Multidimensional latent class Item Response Theory models. R package version 2.10, URL https://cran.r-project.org/web/packages/MultiLCIRT/, 2016. [p144]

A. Bertrand and C. M. Hafner. covLCA: Latent Class Models with Covariate Effects on Underlying and Measured Variables. $\mathrm{R}$ package version 1.0, URL https://cran.rproject.org/web/packages/covLCA/, 2013. [p145]

A. Birnbaum. Some latent trait models and their use in inferring an examinee's ability. In F. M. Lord and M. R. Novick, editors, Statistical Theories of Mental Test Scores, pages 395-479. Addison-Wesley, Reading, MA, 1968. [p145]

R. D. Bock, R. Gibbons, and E. Muraki. Full-information item factor analysis. Applied Psychological Measurement, 12:261-280, 1988. [p139]

W. E. Bonifay. An illustration of the two-tier item factor analysis model. In S. P. Reise and D. A. Revicki, editors, Handbook of Item Response Theory Modeling, pages 207-225. Routledge, 2015. [p139]

L. Cai. A two-tier full-information item factor analysis model with applications. Psychometrika, 75: 581-612, 2010. [p139, 142, 159, 160]

L. Cai, J. S. Yang, and M. Hansen. Generalized full-information item bifactor analysis. Psychological Methods, 16:221-248, 2011. [p139, 142]

G. Celeux and G. Soromenho. An entropy criterion for assessing the number of clusters in a mixture model. Journal of Classification, 13:195-212, 1996. [p143]

P. Chalmers, J. Pritikin, A. Robitzsch, M. Zoltak, K. Kim, C. F. Falk, and A. Meade. mirt: Multidimensional Item Response Theory. $\mathrm{R}$ package version 1.18, URL https://cran.rproject.org/web/packages/mirt/, 2016. [p145]

P. R. Chalmers. mirt: a multidimensional item response theory package for the R environment. Journal of Statistical Software, 48:1-29, 2012. [p145]

A. P. Dempster, N. M. Laird, and D. B. Rubin. Maximum likelihood from incomplete data via the EM algorithm (with discussion). Journal of the Royal Statistical Society B, 39:1-38, 1977. [p142]

S. S. Farivar, W. E. Cunningham, and R. D. Hays. Correlated physical and mental health summary scores for the SF-36 and SF-12 health survey, V. 1. Health and Quality of Life Outcomes, 5:doi: 10.1186/1477-7525-5-54, 2007. [p151]

A. K. Formann. Mixture analysis of multivariate categorical data with covariates and missing entries. Computational Statistics \& Data Analysis, 51:5236-5246, 2007. [p141]

A. K. Formann and T. Kohlmann. Three-parameter linear logistic latent class analysis. In J. A. Hagenaars and A. L. McCutcheon, editors, Applied latent class analysis, pages 183-210. Cambridge University Press, Cambridge, 2002. [p139]

R. D. Gibbons and D. R. Hedeker. Full-information item bi-factor analysis. Psychometrika, 57:423-436, 1992. [p139] 
R. D. Gibbons, R. D. Bock, D. Hedeker, D. J. Weiss, E. Segawa, D. K. Bhaumik, D. J. Kupfer, E. Frank, V. J. Grochocinski, and A. Stover. Full-information item bifactor analysis of graded response data. Applied Psychological Measurement, 31:4-19, 2007. [p139]

L. A. Goodman. Exploratory latent structure analysis using both identifiable and unidentifiable models. Biometrika, 61:215-231, 1974. [p139]

R. K. Hambleton and H. Swaminathan. Item Response Theory: Principles and Applications. Kluwer Nijhoff Publishing, Boston, 1985. [p139]

R. D. Hays, C. D. Sherbourne, and R. M. Mazel. The RAND 36-item health survey 1.0. Health Economics, 2:217-227, 1993. [p151]

E. E. Jang. A framework for cognitive diagnostic assessment. In C. A. Chapelle, Y.-R. Chung, and J. Xu, editors, Towards adaptive CALL: Natural language processing for diagnostic language assessment, pages 117-131. Iowa State University, Ames, IA, 2008. [p144]

M. Jeon, F. Rijmen, and S. Rabe-Hesketh. Flexible item response theory modeling with FLIRT. Applied Psychological Methods, 38:404-405, 2014. [p145]

P. F. Lazarsfeld and N. W. Henry. Latent Structure Analysis. Houghton Mifflin, Boston, 1968. [p139]

G. N. Masters. A Rasch model for partial credit scoring. Psychometrika, 47:149-174, 1982. [p141]

L. Muthén and B. Muthén. Mplus user's guide. Los Angeles, CA, Muthén \& Muthén edition, 2012. [p145]

M. C. Neale, M. D. Hunter, J. N. Pritikin, M. Zahery, T. R. Brick, R. M. Kickpatrick, R. Estabrook, T. C. Bates, H. H. Maes, and S. M. Boker. OpenMx 2.0: Extended structural equation and statistical modeling. Psychometrika, 81(2):535-549, 2016. doi: 10.1007/s11336-014-9435-8. [p145]

G. Rasch. Probabilistic Models for Some Intelligence and Attainment Tests. Danish Intitute for Educational Reserch, Copenhagen, 1960. [p145]

M. D. Reckase. Multidimensional Item Response Theory. Springer, 2009. [p139]

S. P. Reise. The rediscovery of bifactor measurement models. Multivariate Behavioral Research, 47: 667-696, 2012. [p139]

A. Robitzsch, T. Kiefer, A. C. George, and A. Uenlue. CDM: Cognitive Diagnosis Modeling. R package version 5.0-0, URL https://cran.r-project.org/web/packages/CDM/, 2016. [p144]

Y. Rosseel, D. Oberski, J. Byrnes, L. Vanbrabant, V. Savalei, E. Merkle, and et al. lavaan: Latent Variable Analysis. R package version 0.5-20, URL http:/ /lavaan.org, 2015. [p145]

A. A. Rupp and J. L. Templin. Unique characteristics of diagnostic classification models: A comprehensive review of the current state-of-the-art. Measurement: Interdisciplinary Research and Perspectives, 6:219-262, 2008. [p144]

F. Samejima. Estimation of latent ability using a response pattern of graded scores. Psychometrika Monograph Supplement, 34, 1969. [p141]

G. Schwarz. Estimating the dimension of a model. The Annals of Statistics, 6:461-464, 1978. [p143]

A. L. Stewart and J. E. Ware. Measuring Functioning and Well-being. Duke University Press: Durham, 1992. [p150]

K. K. Tatsuoka. Rule space: An approach for dealing with misconceptions based on item response theory. Journal of Educational Measurement, 20:345-354, 1983. [p144]

W. J. Van der Linden and R. K. Hambleton. Handbook of Modern Item Response Theory. Springer, New York, 1997. [p139]

J. Vermunt and J. Magidson. Latent GOLD 4.0 user's guide. Statistical Innovations Inc., Belmont, Massachusetts, 2005. [p145]

M. von Davier. A general diagnostic model applied to language testing data. British Journal of Mathematical and Statistical Psychology, 61:287-307, 2008. [p139, 141]

J. E. Ware and B. Gandek. Overview of the SF-36 health survey and the international quality of life assessment (IQOLA) project. Journal of Clinical Epidemiology, 51:903-912, 1998. [p151] 
J. E. Ware, M. Kosinski, D. M. Turner-Bowker, and B. Gandek. How to Score Version 2 of the SF-12 Health Survey. QualityMetric Incorporated: Lincoln, RI, 2002. [p150]

Silvia Bacci

Department of Economics, University of Perugia

Via A. Pascoli 20, 06123 Perugia

Italy

silvia.bacci@unipg.it

Francesco Bartolucci

Department of Economics, University of Perugia

Via A. Pascoli 20, 06123 Perugia

Italy

francesco.bartolucci@unipg.it 